\title{
UCRL 7463
}

\section{University of California}

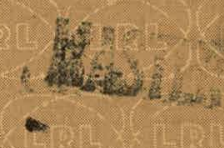

\section{Ernest 0. Lawrence Radiation Laboratory}

\section{CEL: A TIME DEPENDENT, TWO SPACE DIMENSIONAL, COUPLED EULERIAN-LAGRANGE CODE}

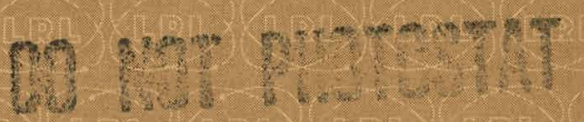

Livermore, California 


\section{DISCLAIMER}

This report was prepared as an account of work sponsored by an agency of the United States Government. Neither the United States Government nor any agency Thereof, nor any of their employees, makes any warranty, express or implied, or assumes any legal liability or responsibility for the accuracy, completeness, or usefulness of any information, apparatus, product, or process disclosed, or represents that its use would not infringe privately owned rights. Reference herein to any specific commercial product, process, or service by trade name, trademark, manufacturer, or otherwise does not necessarily constitute or imply its endorsement, recommendation, or favoring by the United States Government or any agency thereof. The views and opinions of authors expressed herein do not necessarily state or reflect those of the United States Government or any agency thereof. 


\section{DISCLAIMER}

Portions of this document may be illegible in electronic image products. Images are produced from the best available original document. 
This paper was submitted for pubjication prior to the issuance date of this Microcard. Since the U.S.A.E.C. has no evidence that it has been published, the paper is being distributed in Microcard form as a preprint.

\section{UNIVERSITY OF CALIFORNIA \\ Lawrence Radiation Laboratory Livermore, California}

Contract No. W-7405-eng-48

\section{CEL:}

\section{A TIME DEPENDENT, TWO SPACE DIMENSIONAL, COUPLED EULERIAN - LAGRANGE CODE}

W. F. Noh

August 1963

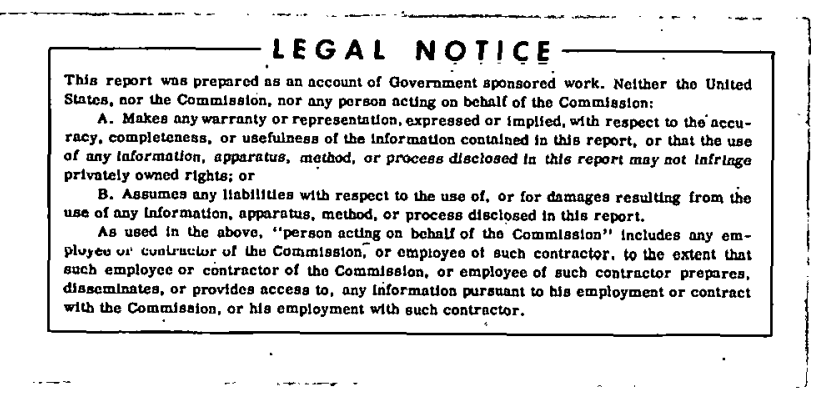


THIS PAGE

\section{WAS INTENTIONALLY LEFT BLANK}


TABLE OF CONTENTS

Page No.

I. Introduction . . . . . . . . . . . . . . $\quad 1$

II. A General Discussion of Hydrodynamical Calculations . 6

III. Description of the CEL Code $\quad . \quad \cdot \quad \cdot \quad \cdot \quad \cdot \quad \cdot \quad 12$

IV. Generalization of the Difference Approximation for Partial Derivatives Over an Arbitrary Region . . . 17

V. Eulerian Difference Equations for Interior Cells of the Mesh . . . . . . . . . . .

VI. The Conservation Form of the Hydrodynamical Equations when the Space Variables $\mathbf{x}$ and $\mathrm{y}$ May have an Arbitrary Velocity Relative to the Fluid Velocity

VII. General Considerations for the Boundary Zone Calculations .. 31

VIII. Boundary Zones and Boundary Sequences . . . . . . $\quad 32$

IX. Boundary Zone Difference Equations for Variables $\rho, \epsilon$, etc. which are Centered in Boundary Zones $\mathrm{C}^{n}(\mathrm{k}, \ell)$.

$X$. Boundary Zone Difference Equations for the Components of Momentum ( $m=\rho u, n=\rho v)$. which are Centered in the Boundary Zones $\mathrm{C}^{n}(\mathrm{k}+1 / 2, \ell+1 / 2) \quad$. . . . . . 40

XI. Lagrange Difference Equations . . . . . . . 42

XII. Discussion and Graphical Results of Several CEL . . . . . . . . . . . 45
Calculations

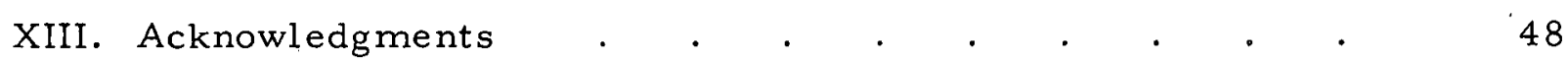


CEL:

A TIME DEPENDENT, TWO SPACE DIMENSIONAL, COUPLED EULERIAN - LAGRANGE CODE*

W. F. Noh

Lawrence Radiation Laboratory, University of California Livermore, California

August 1963

\section{INTRODUCTION}

This article is primarily devoted to the development of a method for calculating the solution of a time-dependent two-space-dimensional Eulerian hydrodynamics problem for a region with an arbitrary polygonal approximating mesh having a general moving (time-dependent) fluid boundary. This new method is the central feature of the CEL (Coupled Euler-Lagrange) calculation.

The CEL code is a time-dependent, two-space-dimensional (compressible, inviscid, non-heat-conducting) hydrodynamics code which enables us to couple an Eulerian approximation of some regions of a fluid with a Lagrange approximation for each of the adjacent regions of the fluid.

The major problem in the CEL method is developing a suitable Eulerian calculation. In CEL the Eulerian boundary is defined by one or more Lagrange lines and is therefore defined by a polygonal line (i.e., a line made up of straight line segments). A moving polygonal line intersecting the fixed Eulerian mesh will create irregular time-dependent boundary zones, and in general these boundary zones can be defined as the union of closed polygons. The problem then for the Eulerian calculation becomes one of approximating the differential equations for a time-dependent, polygonal region in a way that is consistent with the interior equations. We also wish to have an over-all

\section{Ib}


consistency of the difference approximations for the Lagrange and Eulerian regions. This development constitutes the central theme of the article.

In Section II we discuss in some detail the advantages'and limitations of pure Lagrange and pure Eulerian calculations. We find that many problems are more naturally approximated by allowing certain regions to be given an Eulerian description and the remaining regions a Lagrange description. It is for this class of problems that the CEL method has proven most useful.

In Section III we describe the CEL code and the organization of the calculations. We point out that there are some inherent advantages in the CEL method which enable us to approximate fairly complicated fluid regions, and thus we overcome a serious limitation of most calculational methods. As a by-product of the organization of the CEL calculations, we are able to allow different regions of the fluid to be calculated with a time interval $(\Delta t)$ which is characteristic of the particular region, and the details of this procedure are discussed.

In Section IV we develop a generalization of the difference approximation of a partial derivative over an arbitrary region $\mathrm{R}$. We accomplish this by representing a partial derivative, at least approximately, by a line integral taken over the boundary $\partial \mathrm{R}$. Relative to an Eulerian approximating mesh (including boundary zones) the calculation reduces to the problem of evaluation of a line integral over an arbitrary polygon $R$ where the function values are given only at the mesh points (vertices of $\partial R$ ). Consequently we must infer the values of the function on $\partial R$ from the values at these mesh points. The simplest assumption, namely that the function varies linearly between the mesh points reduces the line integral to a finite sum of the average values of the function at the mesh points times appropriate mesh.lengths. We point out that the resulting approximation of partial derivatives is indeed a natural one 
since, in particular, it reduces to the usual approximations when $R$ is a triangle or when $R$ is a quadrilateral. Further we show that the difference approximation of the conservation equation $f_{t}+\underset{\nabla}{f} \underline{U}=0$ leads to a difference equation with the same basic conservation properties. (We make this precise in Theorems 2 and 3.)

We find empirically that the difference equations must retain the conservation property of the Eulerian hydrodynamical differential equations. It is therefore a necessary condition that any difference approximation of partial derivatives must be such that this approximation leads to difference equations which retain this conservation property. We thus find that the generalization of the difference approximation which we employ is completely satisfactory.

In Section $V$ we define the approximating mesh and the space and time centering of the dependent variables including the definition of an artificial viscosity variable $(q)$ (von Neumann and Richtmyer, 1960) which is used to treat automatically the appearance of shocks in the flow. We then define the difference expression for the divergence using the generalizations of Section IV. We give a heuristic description of the physical meaning of the divergence expression and discuss the stability of the difference equations. We then give the complete set of difference equations which hold for the interior points of the mesh and several reasonable options are indicated.

In Section VI we derive the conservation form of the hydrodynamical equations for the situation when the space variables $x$ and $y$ may have an arbitrary velocity relative to the fluid velocity. The governing equations for the Eulerian time dependent boundary zones are a special case of these more general equations. We show that these equations include our Eulerian interior equations when the space variables are independent of time and include the Lagrange equations when the space variables move with the fluid velocity. 
In Section VII we investigate the difference equations of Section VI in the Lagrange limit and determine the time centering of the Jacobian of the transformation (i.e., the transformation of the Lagrange variables to the Eulerian space variables). In Theorem 5 we establish a difference approximation for the Jacobian over a region $R$, and we find an interpretation of the Jacobian as the area of $R$. It is this interpretation (of the Jacobian as an area) that we use when we approximate the more general equations that apply for the boundary zones.

In Section VIII we develop in detail the characterization of a boundary zone and define certain boundary sequences which are associated with a boundary zone. These boundary sequences are needed for the definition of the boundary difference equations. A procedure for the construction of these sequences is sketched and several examples are given.

Now the difference equations are defined on a staggered mesh (that is, the momentum and velocity components are defined at the grid points while the remaining dependent variables (density, energy, etc.) are defined at the center of each cell of the mesh); consequently it is necessary to define boundary zones and the associated boundary sequences for this new mesh. This new mesh is constructed by taking as new lattice points the midpoints of the cells of the original grid. Boundary zones for this staggered grid, of course, are determined in exactly the same way as boundary zones for the original grid; hence these details are omitted.

Having defined boundary zones and the associated sequences, the necessary machinery is at hand to take up the difference equation for the boundary zones.

In Section IX we assume that the appropriate boundary sequences of Section VIII have been determined, and we use the generalized difference 
approximation of the divergence of Section VI. Using the results of the discussion of the Jacobian of Section VII, we proceed to the formulation of the difference equations for some of the generalized differential equations of Section VI, in particular those equations which are nominally centered at the midpoints of boundary zones of the original grid (i. e., the equations for $p ; \epsilon$, etc. ). Special attention is necessary when the area of a boundary zone approaches zero, and this is discussed.

In. Section $\mathrm{X}$ we formulate the difference equations. for the components of momentum $\mathrm{m}=\rho \mathrm{u}$ and $\mathrm{n}=\rho \mathrm{v}$ which are nominally centered at the grid points of the original grid or at the midpoints of the boundary zones of the staggered mesh.

The determination of the boundary pressure at the Eulerian-Lagrange interface is discussed. When we have determined this boundary pressure, the Lagrange phase of the calculation is completely determined.

In Section:XI we define a set of Lagrange difference equations by making use of the general difference approximations of Section IV. We point out that the resulting Lagrange difference equations (for a quadrilateral approximating grid) are just the usual ones that one obtains by evaluating the space derivatives in terms of derivatives with respect to the Lagrange variables. Thus we show that our approach recovers the usual difference equations and at the same time insures an over-all consistency of the Lagrange and Eulerian difference equations.

In Section XII: we present some graphical results of typical CEL problems. 


\section{A. GENERAL DISCUSSION OF HYDRODYNAMICAL CALCULATIONS}

Before proceeding with a description of the CEL code, let us examine the general class of problems in which we are interested and discuss the advantages as well as the limitations of pure Lagrange or pure Eulerian approximations.

In hydrodynamics we wish to solve initial value problems with prescribed boundary conditions. Thus we are given the initial $(t=0)$ state of a fluid in a region $R^{0}=R(x, y, 0)$ of the $x, y$ plane and the external forces acting on the boundary $(\partial R)$ of the fluid for $0 \leq t \leq T$. We are then to determine the state of the fluid and the region $R=R(x, y, t)$ occupied by the fluid for subsequent times $0 \leq \mathrm{t} \leq \mathrm{T}$. In general we deal with several fluids in which case $R=U_{i} R_{i}$ (where $R_{i}=R_{i}(x, y, t)$ is the region occupied by the $\underline{i t h}$ fluid). If we denote by $D_{i}=D_{i}(x, y, t)$ that portion of the boundary of $R_{i}$ which is in the interior of $R\left(i . e_{0}, D_{i}=\partial R_{i} \cap(R-\partial R)\right.$ ), then the lines $D_{i}$ are the positions of the material interfaces of the fluid (called contact discontinuities) and the $D_{i}$ move with the fluids in such a way that the pressure and the normal (to. $D_{j}$ ) component of velocity are continuous. Thus we have the additional task of determining the curves of discontinuity $\mathrm{D}_{\mathrm{i}}$ for $0 \leq \mathrm{t} \leq \mathrm{T}$.

Usually in a Lagrange calculation we approximate the region $R^{0}$ with.a mesh in such a way that the boundary $\partial R^{0}$ and the curves $D_{i}^{0}=D_{i}(x, y, 0)$ correspond to specific Lagrange lines. Since the tangential components of velocity may be discontinuous across contact discontinuities, it is generally necessary to provide special "slip-surface" calculations for those Lagrange lines which define the curves $D_{i}$.

In a Lagrange calculation the mesh points correspond to elements of mass in the fluid, and these mesh points follow the particle paths of the fluid elements. Consequently, the fact that the positions of the boundary $\partial \mathrm{R}$ and of 
the curves $D_{i}$ are automatically determined in the calculation is one of the features that makes a Lagrange approximation so useful. Another important feature (again due to the fact that the approximating grid moves with the fluid) is that the region $R$ is always approximated by the same number of mesh points; thus the initial accuracy of the approximation is in general maintained throughout the calculation. Lagrange calculations have proven to be very accurate as long as the approximating mesh remains regular, and, in general, the number of mesh points needed to approximate a region $R$ accurately is surprisingly small. It has been found that Lagrange calculations are ideal for certain large classes of problems. In particular, in terms of accuracy and economy in the number of mesh points needed, it is practically a requirement to use a Lagrange approximation in following the motion of thin plates or thin ribbons of fluid which move many times their original thickness.

Unfortunately, the very features of the Lagrange method which make it so useful are also the ones which make it totally unsatisfactory for calculating a flow in which turbulence develops or in which contact discontinuities may appear at places in the flow not previously specified. If either a contact discontinuity appears (at other than a Lagrange line which corresponds to one of the curves $D_{i}$ ), or if turbulence develops, the mesh points of the grid will attempt to follow this motion and particles of mass which were initially nearest neighbors in $R^{0}$ no longer remain so physically. Consequently the approximating mesh will become highly distorted, and the calculation.becomes quite inaccurate if, indeed, we are able to proceed with the calculation at all. In most situations in which turbulence develops, the time interval $(\Delta t)$ at which one is required to operate for stability tends to zero. This is due to. the fact that the time interval needed for stability is proportional to the shortest distance separating two Lagrange grid lines and the minimum distance 
between neighboring lines of the grid will in general tend to zero. when the mesh becomes distorted.

Much effort has gone into developing automatic remapping techniques which aim to replace a distorted.mesh with a regular one. This remapping is then done periodically (or even at every time step) and is a us eful technique which allows us to complete or to extend the total time of some Lagrange calculations. However, if a remapping becomes necessary, it essentially means that the fixed mesh of an Eulerian calculation is more appropriate.

A fact which is not generally recognized is that the class of problems which can be solved by the use of such remapping techniques in conjunction with a Lagrange mesh is only slightly larger than the class of problems where no remapping is necessary. The reas on for the failure to enlarge substantially the class of problems which can be solved by the use of remapping techniques.is due to the explicit restriction that only the interior points of the mesh in the subregions $R_{i}$ can be remapped, while the grid points on the boundary $\partial R$, and the grid points on the material interfaces $D_{i}$ must be left unaltered. This most natural requirement (for a Lagrange grid) on the mesh points defining $\partial R$ and the curves $D_{i}$ is simply too restrictive to allow for the general shapes which the region $R$ and the subregions $R_{i}$ may take. Another difficulty (not necessarily involving turbulent-flow problems) develops when a region $R$, which is initially simply connected, subsequently becomes multiply connected. In fact a serious difficulty arises even if a neck develops in some region of $R$. In such cases the distance between separate grid lines of the Lagrange approximating mesh will approach zero (in the neck region), and then the stability condition will force $\Delta$ t to zero. For example, consider the result of squeezing the center of a tooth paste tube. 
It is clear that no amount of rezoning of a Lagrange approximating grid (which keeps the total number of grid lines fixed) will extend the calculation when the region $R$ attempts to become multiply connected. What is required (in general) when rezoning becomes necessary in a Lagrange calculation is to allow the line which defines $\partial R$ to cross the approximating grid lines as it moves and in this way retain the desired number of mesh points in all regions of $R$. Then we automatically allow $R$ to become multiply connected.

A remedy for the difficulties encountered with a Lagrange approximation is provided if we use a multifluid Eulerian approximation (that is, an approximating grid which is fixed and with lines approximating the fluid boundary $\partial \mathrm{R}$ and the material interfaces $D_{i}$, which move relative to this fixed grid). In principle, at least, all hydrodynamical calculations could be approximated by a multifluid Eulerian calculation, but practical considerations limit the class for which it is suitable.

Let us examine the se practical considerations. The procedure for an Eulerian calculation (with, say, a rectangular approximating mesh) is first the determination of a rectangular region $(\mathfrak{R})$ in the $x, y$ plane which is large enough to contain $R$ in its interior for $0 \leq t \leq$ T. We then approximate this rectangular region with a rectangular mesh and, finally, by introducing some auxiliary lines we approximate the boundary $\partial R$ and the curves $D_{i}$.

Now, nature seems to provide for a conservation of difficulties. Even though an Eulerian approximation with its fixed mesh will automatically treat turbulent flow when it appears (and regions are automatically allowed to become multiply connected), the difficulties in an Eulerian calculation lie in determining properly the positions of the (Lagrange) lines which approximate the boundary $\partial R$, and the material interfaces $D_{i}$. 
The curves which approximate $\partial R$ and the $D_{i}$ must move with the fluid and consequently will create irregular, time dependent, boundary zones in the fixed Eulerian mesh. It is not reasonable to expect that we will be able to approximate the differential equations in these irregular boundary zones with the same degree of accuracy that we are able to approximate the differential equations in the interior cells of the mesh. Thus from the standpoint of the accuracy of the calculation at any time $t(0 \leq t \leq T)$, the ratio of the number of interior zones in each subregion $R_{i}$ to the number of boundary zones in each subregion $R_{i}$, which we denote by $A_{i}(t)$, and which is roughly a measure of the accuracy of an Eulerian calculation for the subregion $R_{i}$, should be made as large as possible. The ratios $A_{i}(t)$ can in general be made large (with an approximation mesh of practical size) if the length of $\partial R_{i}$ is small compared to the area of $R_{i}$. If on the other hand, any subregion $R_{i}$ of the region $R$ is such that the length of $\partial R_{i}$ is large compared with the area of $R_{i}$ (as in the case of a subregion which is a long thin ribbon, etc.), then in order for the ratio $A_{j}(t)$ to be large, we must cover $R$ with a fine approximating mesh, and this is normally not practical. Thus for a pure Eulerian calculation to be accurate, we are restricted to those regions $R$ for which all of the ratios $A_{i}(t)$ will be large for all $t, 0 \leq t \leq T$.

Another consideration which is important concerns the number of mesh points in the mesh approximating the large rectangle $R$ which at any given time $0 \leq t \leq T$ lies outside (i.e., the number of mesh points which are exterior points) of the region R. These exterior points are inactive points of the calculation and although it is possible to eliminate the calculations at these exterior points in an Eulerian code, they nevertheless constitute part of the total number of mesh points available in any problem. Hence for a fixed total number of mesh points, the ratios $A_{i}(t)$ will decrease (and hence the accuracy 
of the calculation will decrease) as the number of exterior points increases. If we denote by $\epsilon(t)$ the ratio of points of the mesh which are interior (or on the boundary of $R$ ) to the total number of points of the mesh (which approximate $R$ ), then for the greatest accuracy and economy in any Eulerian calculation $\epsilon(t)$ should be as nearly equal to one as possible. The ratio $\epsilon(t)$ can be thought of as a measure of the efficiency (in terms of accuracy and economy) of an Eulerian calculation.

Generally speaking then, we see that the problems which are most suitable for a pure Eulerian calculation are those in which (for $0 \leq t \leq T$ ) the area of the region $R$ is as close as possible to the area of the largeir rectangular region $\mathscr{R}$ and for which the area of each subregion $R_{i}$ is large compared with the length of the subregion boundary $\partial R_{i}$.

Clearly, many problems are neither ideally suited for a pure Lagrange nor a pure Eulerian calculation but could best be calculated by some combination of Eulerian and Lagrange approximations. Experience has shown that in most problems the region $R$ occupied by the fluids can be decomposed in a natural way into subregions $R_{i}$ (the $R_{i}$ nearly always correpond to subregions which contain different fluids) such that for large intervals in time (t) each subregion $R_{i}$ is most accurately and economically approximated by either a pure Lagrange or a pure Eulerian calculation. That is, we find empirically that most fluids have "natural" Lagrange regions and "natural" Eulerian regions for most of the calculation. In particular, it generally happens that a region $R$ can be accurately approximated by a Lagrange grid from $t=0$ to some time $t_{1}\left(0 \leq t_{1} \leq T\right)$ and for times $t>t_{1}$, the calculation will become inaccurate (or for stability reasons it will be impossible to continue). If at the time $t_{1}$ we allow the se subregions $R_{i}$ of $R$ which are no longer accurately calculated using a Lagrange approximation to be given an Eulerian 
approximation, and the remaining subregions to retain their Lagrange approximations, we find in general that the calculation can be completed.

It is for such classes of problems that either initially or at some later time can benefit by allowing some of the subregions to be approximated by an Eulerian grid and the remaining subregions to be approximated by Lagrange grids that the CEL code was developed. This ability to couple Eulerian and Lagrange calculations in a hydrodynamics code has been found to be most useful, and indeed for many problems it is practically a necessity. For example, problems in which thin plates push on a gas, we find that the thin plate is a natural Lagrange region and that the gas region essentially requires an Eulerian approximation in order to allow for turbulent motion. Similarly, if we wish to calculate the motion of a balloon being inflated or of a soap bubble being formed, we find that the balloon and soap film are natural Lagrange regions, while the inflating gas is a natural Eulerian region.

\section{DESCRIPTION OF THE CEL CODE}

The basic idea in the CEL code is that the boundary $\partial R$, lof the region $R=U_{i} R_{i}$ we wish to approximate) and the curves $D_{i}$ (which separate the subregions $R_{i}$ ) are to be approximated by Lagrange lines. Thus the moving boundaries $\left(\partial R\right.$ and the $\left.D_{i}\right)$ are made to correspond to some Lagrange lines in the Lagrange approximating grids.

A subregion $R_{i}$ which is approximated by the Eulerian mesh will consequently have its boundary $\partial R_{i}$ prescribed by the Lagrange calculations. Thus the Eulerian calculation reduces to a calculation on a fixed mesh having a prescribed moving boundary and the refore constitutes one of the central calculations in the CEL code.

The CEL code consists of a large ${ }^{\text {l }}$ rectangular Eulerian mesh which we denote by $E$, and, depending on the problem we wish to solve, from one to six 
separate Lagrange grids which we denote by $L_{i}(t) i=1,2, \ldots 6$ (i. e., $E$ and the $L_{i}(t)$ are the sets of lattice points which define the Eulerian and the Lagrange grids respectively. Also, the Lagrange grids are denoted as functions of time since their grid points move with the fluids they approximate).

The calculations that are made at each time step in the code are divided into three main parts: Lagrange calculations, Eulerian calculations, and a calculation which couples the Eulerian and Lagrange regions by defining that part of the Eulerian mesh.E which is active and by determining the pressures from the Eulerian region.which acts on the Lagrange boundaries:

We:suppose that we are at the nth time step $\left(t^{n}=\sum_{j}^{m} \Delta t_{j}\right)$ of the CEL calculation and that we wish to advance all quantities by one time step to the time $t^{n+l}$. We assume that the state of the fluids (density, energy, etc.) is known at $t^{\mathrm{n}}$ and also that we know the positions of the Lagrange grids (i. e., we have determined $\left.L_{i}^{n}=L_{i}\left(t^{n}\right)\right)$. In addition, we suppose that we have determined that subset of the Eulerian mesh $\mathrm{E}$, which is interior to, or on the boundary of, those subregions $R_{i}$ having an Eulerian approximation. We denote this suboet of $E$ at $t=t^{n}$ by $E^{n}=E\left(t^{n}\right)$. The calculations for the next time step proceed in the following way.

The first calculation uses the known $\left(t=t^{n}\right)$ state of the Lagrange fluids and the pressures acting on the Lagrange boundaries to solve the Lagrange difference equations for each of the grids $L_{i}^{n}$. The solution to the differences equations gives us the $\mathrm{t}=\mathrm{t}^{\mathrm{n}+1}$ state of the Lagrange fluids and new grid positions $L_{i}^{n+l}$.

Next it is necessary to determine the set $E^{n+1}$, and this is done by one phase of the coupling calculation which uses the new grid positions $L_{i}^{n+l}$. We are then in a position to solve the Eulerian difference approximation equations 
for the $t^{n+1}$ state of the fluid in the region $E^{n+1}$. This is done in the Eulerian phase of the CEL calculation.

Having determined the $t^{n+l}$ state of the Eulerian region, the second phase of the coupling calculation determines the $t^{n+l}$ pressures which act on the boundaries of the Lagrange grids $L_{i}^{n+l}$. We have thus advanced all of the fluid quantities and grid positions to their $t=t^{n+l}$ values and this then completes one basic calculational cycle for one basic time step of the calculation).

The description of the CEL calculation will be completed by giving the "start up" or initial $(t=0)$ state of the fluids and the initial positions of the mesh points for the grids $L_{i}^{0} i=1,2, \ldots, E$ and the subset $E^{0}$. In order to make this more concrete let us consider a simple multifluid problem and give the procedure for approximating this problem on the CEL code.

We consider the region R (see Fig. la) composed of five subregions

(i. e., $R=\bigcup_{i=1}^{i=5} R_{i}$ ) which are each initially $(t=0)$ at rest and at a constant pressure $P_{0}$, with each $R_{.}^{0}$ corresponding to a different gas (i. e., the initial states (except'for pressure) or the equations of state for the different subregions $R_{i}^{0}$ are different). Let $R$ be initially the rectangular region $R^{0}=\{\langle x, y\rangle \mid 0 \leq x \leq a \wedge 0 \leq y \leq b\}$.

The prescribed boundary conditions for $t \geq 0$ are as follows: We consider that the region $R$ has rigid wall boundaries except for the left-hand boundary, which we denote by $\ell=\ell(x, y, t)$ (and which initially is the line $\left.\ell^{0}=\{\langle x, y\rangle \mid x=0 \wedge 0 \leq y \leq b\}\right)$, where a constant pressure $\dot{P}_{1}>P_{0}$ is applied. Thus if $u$ and $v$ are the $x$ and $y$ components of velocity, respectively, then the prescribed boundary conditions are given by:

$$
t \geq 0 \quad v(x, 0, t)=u(a, y, t)=v(x, b, t)=0
$$

and $P_{1} \equiv$ constant on $\ell=\ell(x, y, t)$. 
In order to approximate this problem on the CEL code we must approximate the moving boundary and the interface curves $D_{i}, \ldots D_{4}$ (see Fig. Ia) by Lagrange lines using the Lagrange grids. This is possible by letting the grid $L_{1}^{0}$ approximate $R_{1}^{0}$, $L_{2}^{0}$ approximate $R_{3}^{0}$, and $L_{3}^{0}$ approximate either $R_{4}^{0}$. or $R_{4}^{0} U R_{5}^{0}$. The subregion $R_{2}^{0}$ or the subregions $R_{2}^{0}$ and $R_{5}^{0}$ could then be approximated by the Eulerian mesh $E_{.}^{0}$. To be specific, we let $L_{3}^{0}$ approximate $\mathrm{R}_{4}^{0}$ and we approximate $\mathrm{R}_{2}^{0}$ and $\mathrm{R}_{5}^{0}$ with an Eulerian mesh $\mathrm{E}^{0}$.

In order to define the mesh $\mathrm{E}$ we must first determine a rectangular region $R$ which is large enough to contain the subregion $R_{2}$ and $R_{5}$ in its interior for all $t \geq 0$. Now the boundary pressure $P_{1}$ is greater than the initial pressure $P_{0}$ in $R$, consequently we can expect the boundary $\ell$ to lie always to the right of the y axis. With this assumption on $\ell$ we choose $R=R^{0}$ and we then approximate $\mathbb{R}$ with the Eulerian mesh $E$. The number of mesh points in $E$ and the sets $L_{1}^{0}, L_{2}^{0}, L_{3}^{0}$ will depend on the accuracy we desire up to the maximum permitted in the code.

The multiregion problem we have just considered is a simple example of a problem that would be most difficult to approximate (in a natural way) by a pure Lagrange code. This stems from the fact that the various subregions do not lend themselves to a single approximating mesh in which the contact discontinuities (the curves $D_{1}, \ldots D_{4}$ in $-F i g$. la) correspond to Lagrange lines.

This difficulty in representing even a mildly complicated geometric figure is a serious defect in most Lagrange codes and is a difficulty which occurs in many computational methods in hydrodynamics.

We find that the CEL method of coupling several separate Lagrange grids with an: Eulerian mesh allows a great deal of flexibility in approximating multiregion problems in a most natural way. Indeed, this is one of the most important features of the CEL method. 
Another important feature of the CEL code occurs as a by-product. It is that in a basic computational cycle the Lagrange calculations are done independently for each Lagrange grid $\mathrm{L}_{i}$ and that the Eulerian calculation is done separately as well. These separate calculations enable us to use a separate time interval $\left(\Delta_{i} t\right)$ for each of the Lagrange grids $L_{i}(t)$ all of which may be different from the time interval $\Delta t$ for the Eulerian mesh $E(t)$. We do this in the following.way.

In each basic cycle of the calculation, we determine the maximum $\Delta t$ which.is allowable (from stability considerations) for each of the Lagrange grids $L_{i}(t)$ and for the Eulerian grid $E(t)$. In order to insure the stability of the entire calculation the standard procedure would be to select the minimum of these allowable $\Delta t$ !s and use it for both the Lagrange and Eulerian phases of the next basic calculational cycle. In practice, however, we find that the allowable $\Delta t^{\prime}$ s for the different $L_{i}(t)$ and for $E(t)$ can be quite different, and, in general, the $\Delta t$ for the Eulerian grid $E(t)$ is the largest. Consequently in CEL the $\Delta t$ that is required for stability (at $t=t^{n}$ ) in the Eulerian region $E(t)$ is chosen as the basic time interval for the $(n+1)$ th cycle of the calculation. To insure stability of the Lagrange calculations for the $(n+1)$ th cycle, a time interval is chosen for each grid $L_{j}(t)$ which is "stable for that grid and at the same time is some submultiple of (or equal to) the basic Eulerian $\Delta t$.

Each Lagrange grid is then advanced the appropriate number of time steps so that at the end of the basic calculational cycle all the grids have been advanced to the same total time.

This ability to use different time intervals for the Lagrange and Eulerian grids has proven to be an important feature of the CEL code and has resulted in the saving of much computation time. It.is quite natural that this should be so, since the stability condition on the allowable time interval at a 
point in the fluid is a function of the local sound speed and mesh size at the point (and of the fluid velocity at a point in the Eulerian mesh).

Now, physically the local sound speed (and fluid velocity) can vary considerably in different regions of the fluid, and the mesh size (for accuracy) in general will also be a function of the region being approximated. It is therefore to be expected that the different subregions will have different stability requirements. We find that it is desirable to allow these different regions their "characteristic" time interval in a hydrodynamic calculation.

IV.. GENERALIZATION OF THE DIFFERENCE APPROXIMATION FOR PARTIAL DERIVATIVES OVER A.N ARBITRARY REGION

We shall limit the discussion to two-dimensional cartesian coordinates, the extension to axially symmetric problems being straight-forward with no new difficulties. The differential equations we consider will be approximated by difference equations assuming that the region of the fluid flow in the (x,y)plane is covered by a rectangular mesh. The difference equations for a quadrilateral grid or general polygonal mesh are easily obtained due to the completely general nature of the development, the necessary changes (and the changes required for the axially symmetric case) being indicated at the appropriate places in the text.

There are many equivalent forms of the system of differential equations which characterize the flow of an inviscid non-heat-conducting fluid in Eulerian coordinates, but certain formulations lead to considerably more accurate difference approximations than do others. The differential equations considered here are in conservation form, and these lead to a natural set of difference equations. These difference approximations have proven (empirically) to be quite accurate and generally most satisfactory for a. wide range of two-dimensional problems. 
In the present formulation, momentum is considered.a.fundamental dependent variable and velocity becomes a defined quantity; it is quite important to carry this distinction over to the difference equations. The differential equations in conservation form are given by:

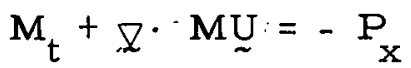

$$
\begin{aligned}
& \mathrm{N}_{\mathrm{t}}+\cdot \nabla \cdot \mathrm{N} \underset{\sim}{\mathrm{U}}=-\mathrm{P}_{\mathrm{y}} \\
& \rho_{\mathrm{t}}+\nabla \cdot \rho \underset{\sim}{\mathrm{U}}=0 \\
& \epsilon_{t}+\underset{\sim}{\nabla} \cdot \underline{\sim}=-P \underset{\sim}{U} \cdot \underset{\sim}{U} \\
& P=P(\epsilon, \rho)
\end{aligned}
$$

where $M=p u$ and $N=p v$ are the $x$ and $y$ components of momentum (per unit volume, $\underset{\sim}{U}=u_{\sim}+v_{\sim}^{j}$ is the velocity, $\rho$ is the density, $\epsilon$ is the internal energy. per unit volume, and $P$ is the pressure.

We note that if $P$ is set equal to zero then (1), (2), and (4) have the same form as (3) and thus express the conservation of these quantities in the absence of a force field.

It has been found empirically that the difference analog of these conservation expressions must also conserve the transport of these quantities algebraically. (This statement is made exact in Theorems 2 and 3.) We . meet this requirement and at the same time obtain a natural generalization of the difference approximation for the partial derivatives (and the expression for the divergence in particular) for an arbitrary covering mesh, by the following theorem.

THEOREM 1: Given a region $R$ with boundary $\partial R$ in the $x, y$ plane and $f, u$, and $v$ as suitable differentiable functions in $R$; then there exists a point: $\left(x_{0}, y_{0}\right)$ in $\mathrm{R}$ for which the following equality holds:

$$
f_{x}\left(x_{0}, y_{0}\right)=\oint_{\partial R} f d y / \oint_{\partial R} x d y,
$$


Similarly, there are points $\left(x_{1}, y_{1}\right),\left(x_{2}, y_{2}\right)$ such that

$$
f_{y} \cdot\left(x_{1}, y_{1}\right)=-\oint_{\partial R} f d x / \oint_{\partial R} x d y
$$

and

$$
\left[(f u)_{x}+(f v)_{y}\right]_{\left(x_{2}, y_{2}\right)}=\left[\oint_{\partial R} f u d y-f v d x\right] / \oint_{\partial R} x d y
$$

in which all contour integrals are taken counter clockwise.

The proof follows immediately from the mean value theorem and Green's theorem. By. Green's theorem,

$$
\iint_{R} f x d x d y=\oint_{\partial R} f d y, \iint_{R} f y d x d y=-\oint_{\partial R} f d x
$$

and

$$
\iint_{R}\left[(f u)_{x}+(f v)_{y}\right] d x d y=\oint_{\partial R} f u d y-f v d x
$$

Now by the mean value theorem, there exist values of $x$ and $y$ in $R$ such that

$$
\begin{aligned}
& f_{x} \oint_{\partial R} x d y=\iint_{R} f_{x} d x d y, \quad f_{y} \oint_{\partial R} x d y=\iint_{R} f y d x d y, \text { and } \\
& {\left[(f u)_{x}+(f v)_{y}\right] \oint_{\partial{ }^{\prime}} x d y=\iint_{R}\left[(f u)_{x}+(f v)_{y}\right] d x d y \text { (where we have }}
\end{aligned}
$$

us ed the equality $\left.\iint_{R} d x d y=\oint_{\partial R} x d y\right)$. Combining thes equalities and solving for $f_{x,}, f_{y}$ and $(f u)_{x}+(f v)_{y}$, we have Eqs. (6), (7) and (8).

We note that (8) could also have been derived by applying the mean value theorem to the divergence theorem, and it, is this application of the divergence theorem that one uses to approximate the divergence for axially symmetric problems.

The regions $R$ we have in mind are, of course, the individual cells of the mesh or the partial boundary zones formed by the intersection of a moving 
Lagrange boundary with a fixed Eulerian grid. Theorem.l enables us to approximate $\mathrm{P}_{\mathrm{x}}, \mathrm{P}_{\mathrm{y}}$ and the divergence terms occurring in. (1) through (4) in a completely automatic way, regardless of the shape of the region.

It is clear that we must know the values of the function on the boundary of the cells of the mesh in order to evaluate the line integrals and to this end we make the following assumptions: Our difference equations enable us to solve for the values of the dependent variables at mesh points, and the definitions of the functions are extended around the boundaries of a cell by linear interpolation between the mesh points, that is, the mesh-point values are joined by straight lines. This reduces the line integrals of our theorem to sums of products of average mesh point values of the function with the appropriate mesh lengths along the boundary of a cell. ${ }^{2}$

Thus (6), (7), and (8) reduce to (see: Fig. 2)

$$
\begin{aligned}
\left(\frac{\Delta f}{\Delta x}\right)_{j}= & \frac{\sum_{i=1}^{N}\left(f_{i+1}+f_{i}\right)\left(y_{i+1}-y_{i}\right)}{\sum_{i=1}^{N}\left(x_{i+1}+x_{i}\right)\left(y_{i+1}-y_{i}\right)} \\
\left(\frac{\Delta f}{\Delta y}\right)_{j}= & \frac{-\sum_{i=1}^{N}\left(f_{i+1}+f_{i}\right)\left(x_{i+1}-x_{i}\right)}{\sum_{i=1}^{N}\left(x_{i+1}+x_{i}\right)\left(y_{i+1}-y_{i}\right)}
\end{aligned}
$$

where the $\mathrm{N}$ vertices of the jth cell of the mesh are numbered counter-clockwise $i=1,2, \ldots N$ and $f_{N+1}=f_{1}, x_{N+1}=x_{1}$, etc. 
To see how reasonable (or natural) the approximations (9) and (10) are let us consider the cases $n=3,4$, that is, over triangular and quadrilateral regions.

In case $\mathrm{n}=3$ the standard approximations to the partial derivatives $\partial f / \partial x$ and $\partial f / \partial y$ are obtained byipassing a plane through the three vertices and assigning to $\Delta f / \Delta x$ and $\Delta f / \Delta y$ the values obtained by evaluating $\partial f / \partial x$ and $\partial f / \partial y$ at some point on this surface. Now a plane passing through the three points $(x, y, f)_{i} i=1,2,3$ has the form $f=A x+B y+C$, where $A, B$, and $C$. are constants. Thus $f_{x}=A, f_{y}=B$ and it is easily verified that $A$ is just (9) and $B$ is $(10)$.

In the case $\mathrm{n}=4$ a straight-forward approximation is obtained by making a change of variable $x=x(a, b), y=y(a, b)$ such that the quadrilateral $(x, y)$ i $i=1,2,3,4$ is the map of a square $(a, b)_{i} i=1,2,3,4$ in the $a, b$ plane with sides parallel to the $a$ and $b$ axis (Fig, 3). Then one may. evaluate $f_{x}$ and $f_{y}$ in terms of derivatives with respect to $a$ and $b$; i.e., $f_{x}=(1 / J)\left(f_{a} y_{b}-f_{b} y_{a}\right)$, $f_{y}=-(1 / J)\left(f_{a} x_{b}-f_{b} x_{a}\right)$ where $J$ is the Jacobian $x_{a} y_{b}-x_{b} y_{a}$ of the transformation. Using the natural approximations for derivatives with respect to $a$ and $b$ for a square in the $a, b$, plane, i.e.,

$$
f_{a}=\frac{\left(f_{2}+f_{3}\right)-\left(f_{4}+f_{1}\right)}{2\left(a_{2}-a_{1}\right)}, f_{b}=\frac{\left(f_{3}+f_{4}\right)-\left(f_{1}+f_{2}\right)}{2\left(b_{3}-b_{2}\right)},
$$

it is easy to verify that the resulting approximations of $f_{x}$ and $f_{y}$ are just (9) and $(10)$.

We now wish to establish a further property of our difference approximations by considering the conservation equation

$$
f_{t .}+(f u)_{x}+(f v)_{y}=0
$$

and the corresponding conservation difference equation obtained by úsing (11). 
We note that the conservation equation (12) has the following property. THEOREM 2: If $f=0$ on the boundary $\partial D$ of a domain $D$ in the $x$; $y$ plane and $f_{t}+(f u)_{x}+(f v)_{y}=0$ in $D$, then $\iint_{D} f d x d y=$ constant.

PROOF: This follows immediately from our hypothesis that $f=0$ on $\partial \mathrm{D}$ and the divergence theorem, since

$\frac{\partial}{\partial t} \iint_{D} f d x d y=\iint_{D} f_{t} d x d y=-\iint_{D}\left[(f u)_{x}+(f v)_{y}\right] d x d y=-\oint_{\partial D}[(f u) d y-(f v) d x]=0$.

The corresponding difference statement is provided by the following theorem: Letting $f_{j}^{n}$ denote the average value of $f$ in the $j$ th cell at the nth $^{\prime}$ time step, we approximate $f_{t}$ by

$$
\frac{f_{j}^{n+1}-f_{j}^{n}}{\Delta t}
$$

and using (11) our differences approximation of (12) is given by

$$
f_{j}^{n+l}=f_{j}^{n}-\Delta t\left[\frac{\Delta(f(1)}{\Delta x}+\frac{\Delta(f v)}{\Delta y}\right]_{j}^{n} \text {, }
$$

and we prove the following:

THEOREM 3: Let $T$ denote the network of lines comprising the finite

difference mesh. If $f_{j}^{n}=0$ on the boundary $\partial \mathrm{T}$ of the mesh $\mathrm{T}$, then

$$
\sum_{j} f_{j}^{n} A_{j}=\text { constant }
$$

(as a function of $n$ ) where $A_{j}$ is the area of the jth cell, and the sum is extended over all cells of $T$.

PROOF: Our result will be established if we can show that

$$
\sum_{j} f_{j}^{n+l} A_{j}=\sum_{j} f_{j}^{n} A_{j}
$$


Thus from (13) we wish to show that

$$
\sum_{j}\left[\frac{\Delta(f u)}{\Delta x}+\frac{\Delta(f v)}{\Delta y}\right]_{j}^{n} A_{j}=0
$$

Substituting from (1l), we see that the above equation is equivalent to

$$
\sum_{j} \frac{1}{2} \sum_{i}^{N}\left\{\left[(f u)_{i+1}+(f v)_{i}\right]\left(y_{i+1}-y_{i}\right)-\left[(f v)_{i+1}+(f v)_{i}\right]\left(x_{i+1}-x_{i}\right)\right\}=0
$$

(since the denominator $\sum_{i=1}^{N}\left(x_{i+1}+x_{i}\right)\left(y_{i+1}-y_{i}\right)$ of $(11)$ is just $\left.2 A_{j}\right)$. Now (14) does indeed hold, since, in summing over all cells, each side of an interior cell is traversed twice in opposite directions and thus cancel in pairs. Thus only terms on the boundary $\partial \mathrm{T}$ of $\mathrm{T}$ remain and these vanish because, by hypothesis, the $f_{j}^{n}$ vanish on the boundary.

Having examined the reasonableness of (9), (10) and (11), we now pass to the particular case of a rectangular mesh and the difference approximations of (1) through (5). We must distinguish between regular interior cells and the irregular boundary cells formed by the intersection of the fluid boundary with the grid lines.

\section{EULERIAN DIFFERENCE EQUATIONS FOR INTERIOR CELLS OF THE $\mathrm{MESH}$}

Our rectangular mesh is defined by the lines $\mathrm{x}_{\mathrm{k}} ; \mathrm{k}=0,1, \ldots, \mathrm{y}_{\ell} ; \ell=0$, $1,2, \ldots$; and their intersections give the lattice points $\mathrm{x}=\mathrm{x}_{\mathrm{k}} ; \mathrm{y}=\mathrm{y}_{\ell} ; \mathrm{k}, \ell=0$, $1,2, \ldots$ (see Fig. 4).

Certain of the dependent variables (momentum and velocity components) are most naturally defined at the lattice points of the grid and at $(n+1 / 2) \Delta t$ points in time. The remainder $(\rho, \epsilon$, and $p)$ are defined at the midpoints

$$
\left(x_{k+1 / 2, \ell+1 / 2}, y_{k+1 / 2, \ell+1 / 2}\right)=\left(\frac{x_{k+1}+x_{k}}{2}, \frac{y_{\ell+1}+y_{\ell}}{2}\right)
$$


of the cells and at integral values $n \Delta t$ in time. We denote

$$
f\left[x_{k, \ell}, y_{k, \ell},(n+l / 2) \Delta t\right]
$$

by $f_{k, l}^{n+1 / 2}$

and

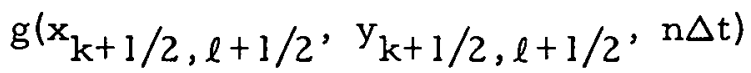

by

$$
g_{k+1 / 2, \ell+1 / 2}^{n} \text {. }
$$

In particular we solve for $M_{k, l}^{n+1 / 2}, N_{k, l}^{n+1 / 2}, u_{k, l}^{n+1 / 2}, v_{k, l}^{n+1 / 2}$, and $\rho_{\mathrm{k}+1 / 2, \ell+1 / 2}^{\mathrm{n}}, \epsilon_{\mathrm{k}+1 / 2, \ell+1 / 2}^{\mathrm{n}}, \mathrm{P}_{\mathrm{k}+1 / 2, \ell+1 / 2}^{\mathrm{n}}$. In addition we solve for a mixed variable $q_{k+1 / 2, \ell+1 / 2}^{n+1 / 2}$ which is an artificial viscosity (von Neumann and Richtmyer, 1950) and is necessary (unless shock fitting or other techniques are used) in the difference equations to represent shock discontinuities properly. The artificial viscosity (q) acts to spread a shock over a fixed number of cells in such a way that the function values vary continuously through the region of the shock and satisfy the Rankine-Hugoniot conservation relations. We shall consider $\mathrm{q}$ to be defined by

$$
\varphi=C_{0}^{2} L^{2} \dot{P}\left\{\begin{array}{lll}
{[\underset{\sim}{\nabla}, \underset{\sim}{U}]^{2}} & \text { if } \underset{\sim}{\nabla} \cdot \underset{\sim}{U} \leq 0 \\
& \text { if } \underset{\sim}{\nabla} \cdot \underset{\sim}{U}>0
\end{array} .\right.
$$

where $C_{0}^{2}$ is a constant weakly dependent on the equation of state of the materials ( $C_{0}^{2}$ is approximately equal to one), and $L$ is a length appropriate to the cell in which $q$ is calculated. $\left(L^{2}\right.$ is of the order of $(\Delta x)^{2}+(\Delta y)^{2}$.)

For functions defined at the mid-points of the cell, we make the following definitions based on (11).

\section{Definition I}

$\left.\begin{array}{l}\left.\text { (i) } \begin{array}{l}u_{k+1, \ell+1 / 2}^{n+1 / 2}=1 / 2\left(u_{k+1, \ell}^{n+1 / 2}+u_{k+1, \ell+1}^{n+1 / 2}\right. \\ \text { (ii) } v_{k+1 / 2, \ell+1}^{n+1 / 2}=1 / 2\left(v_{k+1, \ell+1}^{n+1 / 2}+v_{k, \ell+1}^{n+1 / 2}\right.\end{array}\right)\end{array}\right\}$ i. e., average of known values 
(iii) (fu $\Delta y)_{k+1, \ell+1 / 2}^{n+1 / 2}=\left\{\begin{array}{l}f_{k+1 / 2, \ell+1 / 2}^{n} u_{k+1, \ell+1 / 2}^{n+1 / 2}\left(y_{\ell+1}-y_{\ell}\right) \text { if } u_{k+1, \ell+1 / 2}^{n+1 / 2} \geq 0 \\ f_{k+3 / 2, \ell+1 / 2}^{n} u_{k+1, \ell+1 / 2}^{n+1 / 2}\left(y_{\ell+1}-y_{\ell}\right) \text { if } u_{k+1, \ell+1 / 2}^{n+1 / 2} \leq 0\end{array}\right.$

(iv) $(f v \Delta x)_{k+1 / 2, \ell+1}^{n+1 / 2}=\left\{\begin{array}{l}f_{k+1 / 2, \ell+1 / 2}^{n} v_{k+1 / 2, \ell+1}^{n+1 / 2}\left(x_{k+1}-x_{k}\right) \text { if }\left(v_{k+1 / 2 ; \ell+1}^{n+1 / 2}\right) \geq 0 \\ f_{k+1 / 2, \ell+3 / 2}^{n} v_{k+1 / 2, \ell+1}^{n+1 / 2}\left(x_{k+1}-x_{k}\right) \text { if }\left(v_{k+1 / 2, \ell+1}^{n+1 / 2}\right) \leq 0\end{array}\right.$.

(v) $[\underset{\sim}{\nabla} f \underset{\sim}{U}]_{k+1 / 2, \ell+1 / 2}^{n+1 / 2}=\frac{(f u \Delta y)_{k+1, \ell+1 / 2}^{n+1 / 2}+(f v \Delta x)_{k+1 / 2, \ell+1}^{n+l / 2}-(f u y)_{k, \ell+1 / 2}^{n+1 / 2}-(f v \Delta x)_{k+1 / 2, \ell}^{n+l / 2}}{\left(s_{k+1}+x_{k}\right)\left(y_{\ell+1}-y_{\ell}\right)^{\prime}}$.

Before proceeding to the difference equations a word is :in order regarding the definition of the divergence $[\underset{\sim}{\nabla} \cdot \underset{\sim}{\mathrm{U}}]_{\mathrm{k}+1 / 2, \ell+1 / 2}^{\mathrm{n}+1 / 2}$

Physically it has an intuitive meaning, for if $f$ is considered as a step function, i.e., constant over a cell, then the tests on the direction of the velocity normal to the sides of a cell dictate which values of $f$ are transported in or out of the cell in one time step. (It might seem more natural to transport the average value of $f$ across a cell side, but such action leads to mildly unstable difference equations.) The question of the stability of the difference equations leads to somewhat complicated algebraic equations and will not be discussed here; however, the two limiting cases $\underset{\sim}{U} \cdot \underset{\sim}{U} \gg C^{2}$, and $C^{2} \gg \underset{\sim}{U} \underset{\sim}{U}$ (where $C$ is the local sound speed) (again, not discussed) can easily be shown to imply that the following inequalities must hold for each cell of the mesh: $\Delta t<\Delta x \Delta y /[\Delta y|u|+\Delta x|v|]$ and $\Delta t<\min (\Delta x, \Delta y) / C$. For the general case the following restriction has been found satisfactory in practice:

$$
\Delta t \leq \frac{\Delta x \Delta y}{\Delta y|u|+\Delta x|v|+\max (\Delta x, \Delta y) C}
$$


Using Definition I, then, the difference equations corresponding to (3), (4), (5), and (15) are given by

$$
\rho_{\mathrm{k}+1 / 2, \ell+1 / 2}^{\mathrm{n}+1}=\rho_{\mathrm{k}+1 / 2, \ell+1 / 2}^{\mathrm{n}}-\Delta \mathrm{t}[\underset{\sim}{\nabla} \cdot \rho \underset{\sim}{\mathrm{U}}]_{\mathrm{k}+1 / 2, \ell+1 / 2}^{\mathrm{n}+1 / 2}
$$

$\mathrm{q}_{\mathrm{k}+1 / 2, \ell+1 / 2}^{\mathrm{n}+1 / 2}=\mathrm{c}_{0}^{2} \ell_{\mathrm{k}+1 / 2, \ell+1 / 2}^{2}\left(\rho_{\mathrm{k}+1 / 2, \ell+1 / 2}^{\mathrm{n}+1}+\rho_{\mathrm{k}+1 / 2, \ell+1 / 2}^{\mathrm{n}}\right)\left\{\begin{array}{c}\left([\underset{\sim}{\mathrm{N}} \underset{\sim}{\mathrm{U}}]_{\mathrm{k}+1 / 2, \ell+1 / 2}^{\mathrm{n}+1 / 2}\right)^{2} \\ 0\end{array}\right.$

$$
\begin{aligned}
& \text { if }[\underset{\sim}{\nabla} \cdot \underset{\sim}{\mathrm{U}}]_{\mathrm{k}+1 / 2, \ell+1 / 2}^{\mathrm{n}+1 / 2}<0 \\
& \text { If }[\underset{\sim}{\nabla} \cdot \underset{\sim}{\mathrm{U}}]_{\mathrm{k}+1 / 2, \ell+1 / 2}^{\mathrm{n}+1 / 2}>0
\end{aligned}
$$

$$
\begin{aligned}
& \epsilon_{\mathrm{k}+1}^{\mathrm{n}+1} / 2, \ell+1 / 2=\epsilon_{\mathrm{k}+1 / 2, \ell+1 / 2}^{\mathrm{n}}-\Delta \mathrm{t}\left\{[\underset{\sim}{\nabla} \cdot \epsilon \underset{\sim}{\mathrm{U}}]_{\mathrm{k}+1 / 2, \ell+1 / 2}^{\mathrm{n}+1 / 2}+\left(\mathrm{P}_{\mathrm{k}+1}^{\mathrm{n}+1 / 2}, \ell+1 / 2\right.\right. \\
& \left.\left.+\mathrm{q}_{\mathrm{k}+1 / 2, \ell+1 / 2}^{\mathrm{n}+1 / 2}\right)[\underset{\sim}{\mathrm{U}}]_{\mathrm{k}+1 / 2, \ell+1 / 2}^{\mathrm{n}+1 / 2}\right\} \\
& P_{k+1 / 2, \ell+1 / 2}^{n+1}=P\left(\rho_{k+1 / 2, \ell+1 / 2}^{n+1}, \frac{n+1}{k+1 / 2, \ell+1 / 2}\right) .
\end{aligned}
$$

The value $\mathrm{P}_{k+1 / 2, \ell+1 / 2}^{\mathrm{n}+1 / 2}$ in (18) is ordinarily obtained in one iteration by using $P_{k+1 / 2, \ell+1 / 2}^{n}$ as a first guess and then averaging the result of the first guess with $\mathrm{P}_{\mathrm{k}+1 / 2, \ell+1 / 2}^{\mathrm{n}}$ for the final estimate.

We next consider the variables $(M=\rho u$. and $N=\rho v)$ centered at the lattice points $\left(x_{k}, y_{\ell}\right)$. As before we make the following definitions based on (9), (10), (11) and the numbering of Fig. 5. Define $x_{1}=1 / 2\left(x_{k}+x_{k+1}\right)$; $y_{1}=1 / 2\left(y_{\ell-1}+y_{\ell}\right)$, etc.

Also,

$$
\begin{aligned}
& u_{1}=1 / 4\left(u_{k, \ell-1}+u_{k+1, \ell-1}+u_{k+1, \ell}+u_{k, \ell}\right) \\
& v_{1}=1 / 4\left(v_{k, \ell-1}+v_{k+1, \ell-1}+v_{k+1, \ell}+v_{k, \ell}\right) .
\end{aligned}
$$

Let

$$
\bar{P}^{n}=P^{n}+q^{n-1 / 2}
$$




\section{$\underline{\text { Definition II }}$}

(i) $\left(\frac{\Delta \overline{\mathrm{P}}}{\Delta \mathrm{x}}\right)_{\mathrm{k}, \ell}^{\mathrm{n}} ;=\frac{1}{2} \frac{\overline{\mathrm{P}}_{1}^{\mathrm{n}}+\overline{\mathrm{P}}_{2}^{\mathrm{n}}-\overline{\mathrm{P}}_{3}^{\mathrm{n}}-\overline{\mathrm{P}}_{4}^{\mathrm{n}}}{\mathrm{x}_{1}-\mathrm{x}_{4}}$

(ii) $\left(\frac{\Delta \overline{\mathrm{P}}}{\Delta \mathrm{y}}\right)_{\mathrm{k}, \ell}^{\mathrm{n}}=\frac{1}{2} \frac{\overline{\mathrm{P}}_{2}^{\mathrm{n}}+\overline{\mathrm{P}}_{3}^{\mathrm{n}}-\overline{\mathrm{P}}_{1}^{\mathrm{n}}-\overline{\mathrm{P}}_{4}^{\mathrm{n}}}{\mathrm{y}_{2}-\mathrm{y}_{1}}$

$(\text { iii })^{3}(f u \Delta y)_{k+1 / 2, l}^{n-1 / 2}=\left(y_{2}-y_{1}\right) \frac{\left(u_{5}^{n-1 / 2}+u_{6}^{n-1 / 2}\right)}{2} \cdot\left\{\begin{array}{l}f_{5}^{n-1 / 2} \text { if }\left(u_{5}^{n-1 / 2}+u_{6}^{n-1 / 2}\right) \geq 0 \\ f_{6}^{n-1 / 2} \text { if }\left(u_{5}^{n-1 / 2}+u_{6}^{n-1 / 2}\right)<0\end{array}\right.$

(iv) ${ }^{4}(f v \Delta x)_{k, \ell+1 / 2}^{n-1 / 2}=\left(x_{2}-x_{3}\right) \frac{\left(v_{5}^{n-1 / 2}+v_{7}^{n-1 / 2}\right)}{2} \cdot\left\{\begin{array}{l}f_{5}^{n-1 / 2} \text { if }\left(v_{5}^{n-1 / 2}+v_{7}^{n-1 / 2}\right) \geq 0 \\ f_{7}^{n-1 / 2} \text { if }\left(v_{5}^{n-1 / 2}+v_{7}^{n-1 / 2}\right)<0\end{array}\right.$

(v) $[\underset{\sim}{\nabla} \cdot \underset{\sim}{U}]_{k, \ell}^{n-1 / 2}=\frac{(f u \Delta y)_{k+1 / 2, \ell}^{n-1 / 2}+(f v \Delta x)_{k, \ell+1 / 2}^{n-1 / 2}-(f u \Delta y)_{k-1 / 2, \ell}^{n-1 / 2}-(f v \Delta x)_{k, \ell-1 / 2}^{n-1 / 2}}{\left(x_{1}-x_{4}\right)\left(y_{2}-y_{1}\right)}$.

The difference equations of (1) and (2) are then given by

$$
\begin{aligned}
& \mathrm{M}_{\mathrm{k}, \ell}^{\mathrm{n}+1 / 2}=\mathrm{M}_{\mathrm{k}, \ell}^{\mathrm{n}-1 / 2}-\Delta \mathrm{t}\left\{[\underset{\sim}{\nabla} \cdot \underset{\sim}{\mathrm{M}}]_{\mathrm{k}, l}^{\mathrm{n}-1 / 2}+\left(\frac{\Delta \overline{\mathrm{P}}}{\Delta \mathrm{x}}\right)_{\mathrm{k}, \ell}^{\mathrm{n}}\right\} \\
& N_{k, l}^{n+1 / 2}=N_{k, l}^{n-1 / 2}-\Delta t\left\{\left[\underset{\sim}{\nabla} \cdot N_{\sim}^{U}\right]_{k, l}^{n-1 / 2}+\left(\frac{\Delta \bar{P}}{\Delta y}\right)_{k, l}^{n}\right\}
\end{aligned}
$$

and $\mathrm{u}$ and $\mathrm{v}$ are defined by

$$
\begin{aligned}
& u_{k, l}^{n+1 / 2}=\frac{M_{k, l}^{n+1 / 2}}{\rho_{k, l}^{n}} \\
& \dot{v}_{k, l}^{n+1 / 2}=\frac{N_{k, l}^{n+1 / 2}}{\rho_{k, l}^{n}}
\end{aligned}
$$


where

$$
\rho_{\mathrm{k}, \ell}^{\mathrm{n}}=\frac{1}{4}\left[\rho_{\mathrm{k}+1 / 2, \ell+1 / 2}^{\mathrm{n}}+\rho_{\mathrm{k}-1 / 2, \ell+1 / 2}^{\mathrm{n}}+\rho_{\mathrm{k}-1 / 2, \ell-1 / 2}^{\mathrm{n}}+\rho_{\mathrm{k}+1 / 2, \ell-1 / 2}^{\mathrm{n}}\right]
$$

Clearly equations (9), (10), and (11) were hardly necessary in the development of our interior difference equations, as the regularity of a rectangular mesh would lead to these equations in a most natural way. This development was followed to exhibit the "naturalness" of (9), (10), and (11) and to establish the procedure neressary to obtain differ arbitrary approximation mesh (i.e., one not necessarily rectangular). It is also valuable to know that the interior difference equations are just special cases of the more general boundary equations which we treat in the next section.

VI. THE CONSER VATION FORM OF THE HYDRODYNAMICAL EQUATIONS WHEN THE SPACE VARIABLES X AND Y MAY HAVE AN ARBITRARY V.ELOCITY RELATIVE TO THE FLUID VELOCITY

Before proceeding to the difference equations for the boundary zones, we must consider the more general set of differential equations that rharacterize the fluid flow relative to space variables $x$ and $y$ which are in neither Eulerian nor Lagrange coordinates and which may move relative to the fluid in an arbitrary way. In our particular case the boundary of the fluid is a Lagrange line (in that it moves with the fluid) and moves across the stationary Eulerian gridi hence in the houndary region we have no misnd ayatom.

We consider $x$ and $y$ as functions of the independent variables $a, b$, and $\mathrm{t}$ and let $\omega=\mathrm{u}-\mathrm{x}_{\mathrm{t}}, \sigma=\mathrm{v}-\mathrm{y}_{\mathrm{t}}$, and $\underset{\sim}{\Omega}=\omega \underset{\sim}{\mathrm{i}}+\sigma \underset{\sim}{\mathrm{j}}$. It can be shown that the total time derivative is then given by

$$
\dot{\mathrm{F}}=\mathrm{F}_{\mathrm{t}}+\omega \mathrm{F}_{\mathrm{x}}+\sigma \mathrm{F}_{\mathrm{y}}=\mathrm{F}_{\mathrm{t}}+\underset{\sim}{\Omega} \cdot \underset{\sim}{\nabla} \mathrm{F} \text {. }
$$

We note this :reduces to the Eulerian time derivative for $x_{t}=y_{t}=0$ and to $F_{t}$ as it should in the Lagrange limit $x_{t}=u, y_{t}=v$. 
The hydrodynamic equations, as usual, are given : by

$$
\begin{aligned}
& \dot{\mathrm{u}}+\frac{1}{\rho} \mathrm{P}_{\mathrm{x}}=0 \\
& \dot{\mathrm{v}}+\frac{1}{\rho} \mathrm{P}_{\mathrm{y}}=0 \\
& \dot{\rho}+\rho \underset{\sim}{ } \cdot \underset{\sim}{U}=0 \\
& \dot{\mathrm{e}}+\frac{1}{\rho} \mathrm{P} \underset{\sim}{\nabla} \cdot \underset{\sim}{\mathrm{U}}=0 \\
& P(\rho, e)=0
\end{aligned}
$$

where e is the internal energy per unit mass.

The set (23) is not in a conservation form consistent with the set (1) through (4) which was used for the interior points of the mesh.. We shall use the identity (26) of Theorem 4, below, to bring the equations into the required. form.

THEOREM 4. If $J=\left(\frac{x, y}{a, b}\right)$ denotes the Jacobian of $x$ and $y$ with respect to $a$ and $b, \underset{\sim}{\Omega}=\omega \underset{\sim}{i}+\sigma j$, and if $\rho$ satisfies the equation $\dot{\rho}+\rho \underset{\sim}{\nabla} \cdot \underset{\sim}{U}=0$ then

$$
\begin{aligned}
& \underset{\sim}{\nabla} \cdot \underset{\sim}{U} \equiv \underset{\sim}{\nabla} \cdot \underset{\sim}{\Omega}+\frac{J_{t}}{J} \\
& \rho_{t}+\rho \frac{J_{t}}{J}+\underset{\sim}{\nabla} \cdot \rho \underset{\sim}{\Omega}=0
\end{aligned}
$$

and for any sufficiently differentiable function $f$

$$
\rho \dot{f} \equiv(\rho f)_{t}+(\rho f) \frac{J_{t}}{J}+\underset{\sim}{\nabla} \cdot(\rho f) \Omega .
$$

PROOF:

$$
\underset{\sim}{\nabla} \cdot \underset{\sim}{\Omega}=(\mathrm{u}-\dot{\mathrm{x}})_{\mathrm{x}}+(\mathrm{v}-\mathrm{y})_{\mathrm{y}}=\underset{\sim}{\nabla} \cdot \underset{\sim}{\mathrm{U}}-\left\{\left(\mathrm{x}_{\mathrm{t}}\right)_{\mathrm{x}}+\left(\mathrm{y}_{\mathrm{t}}\right)_{\mathrm{y}}\right\}
$$

Now making use of the results of implicit differentiation

$$
\text { (i.e., : } \left.f_{x}=\frac{1}{J}\left\{\left(\frac{f, y}{a, b}\right)\right\} \text { and } f_{y}=\frac{-1}{J}\left\{\left(\frac{f, x}{a, b}\right)\right\}\right) \text {. }
$$


we have

$$
\left(x_{t}\right)_{x}+\left(y_{t}\right)_{y}=\frac{1}{J}\left\{\left(\frac{x_{t} \cdot y}{a, b}\right)+\left(\frac{x, y_{t}}{a, b}\right)\right\}=\frac{J_{t}}{J}
$$

Hence

$$
\underset{\sim}{\nabla} \cdot \underset{\sim}{\Omega}=\underset{\sim}{\nabla} \cdot \underset{\sim}{\mathrm{U}}-\frac{\mathrm{J}}{\mathrm{J}}
$$

which:is (24). Now (25) follows immediately from this and (22) as

$$
\rho_{\mathrm{t}}+\rho \frac{\mathrm{J}}{\mathrm{J}}+\underset{\sim}{\nabla} \rho \underset{\sim}{\Omega}=\rho_{\mathrm{t}}+\underset{\sim}{\Omega} \cdot \underset{\sim}{(\underset{\nabla}{\rho})+\rho}\left[\frac{\mathrm{J} t}{\mathrm{~J}}+\underset{\sim}{\nabla} \cdot \underset{\sim}{\Omega}\right]=\dot{\rho}+\rho \underset{\sim}{\nabla} \cdot \underset{\sim}{\mathrm{U}},
$$

which is zero by hypothesis. I Iikewise, on expanding the right-hand side of (26) and using (22), (24), and (25), we have

$$
\begin{aligned}
(\rho f)_{t}+(\rho f) \frac{J_{t}}{J}+\underset{\sim}{\nabla} \cdot(\rho f) \underset{\sim}{\Omega} & \left.=\rho f f_{t}+f \rho_{t}+\rho \underset{\sim}{\Omega} \cdot \underset{\sim}{\nabla f}\right)+f \underset{\sim}{\nabla} \cdot \rho \underset{\sim}{\Omega}+\rho f \frac{J_{t}}{J} \\
& =\rho\left[f_{t}+\underset{\sim}{\Omega}(\underset{\sim}{\nabla})\right]+f\left[\rho_{t}+\rho \underset{t}{J}+\underset{\sim}{\nabla} \cdot \rho \underset{\sim}{\Omega}\right]=\rho \dot{f} .
\end{aligned}
$$

We then obtain the equations in conservation form by multiplying the first, second, and third equations of (23) by $\rho$ and using the identiy (26) ((25) being the density equation in conservation form).

$$
\begin{aligned}
& M_{t}+M \frac{J_{t}}{J}+\underset{\nabla}{M} \underset{\sim}{\Omega}+P_{x}=0 \\
& N_{t}+N \frac{J_{t}}{J}+\underset{\sim}{\nabla} \cdot \underset{\sim}{N}+P_{\dot{y}}=0 \\
& \rho_{t .}+\rho \frac{J_{t}}{J}+\nabla \cdot \rho \Omega=0 \\
& \epsilon_{t}+\epsilon \frac{\mathrm{J}}{\mathrm{t}}+\underset{\sim}{\nabla} \cdot \epsilon \underset{\sim}{\Omega}+\underset{\sim}{\nabla} \cdot \underset{\sim}{U}=\underline{\rho} \\
& P=P(\dot{\rho}, \epsilon) \text {. }
\end{aligned}
$$

In particular, we note that if $\mathrm{x}$ and $\mathrm{y}$ are not functions of time then $\mathrm{J}_{\mathrm{t}}=0, \underset{\sim}{\Omega}=\underset{\sim}{\mathrm{U}}$, and we have the Eulerian equations (1) through (5). Similarly, if $\mathrm{x}$ and $\mathrm{y}$ move with the fluid velocity $\left(\mathrm{x}_{\mathrm{t}}=\mathrm{u}, \mathrm{y}_{\mathrm{t}}=\mathrm{v}\right.$, thus $\left.\underset{\sim}{\Omega}=0\right)$ then (27) through (31) reduce to our Lagrange equations in conservation form. 
The boundary zone difference equations are a particularly simple case of (27) through (31) in that $\underset{\sim}{\Omega}$ reduces to $\underset{\sim}{U}$ on the sides of a boundary cell which are part of the Eulerian grid, and $\underset{\sim}{\Omega}$ reduces to zero on the moving Lagrange boundary. In view of this, we will define the difference approximation of (27) through (31) for just this special case. Before proceeding, to the general equations, let us consider the differencing of the first two terms in each of the equations (27) through (30).

\section{GENERAL CONSIDERATIONS FOR THE BOUNDARY ZONE CALCULATIONS}

We consider the special case of the Lagrange limit (i. e., $\Omega=0$ ) of the mass - conservation equation (29).

$$
\rho_{t}+\rho \cdot \frac{J_{t}}{J}=0
$$

Differencing (32) at some point $\left(x_{i}, y_{i}\right)$ in a region $R$, we have

$$
\rho_{i}^{n+1}=\rho_{i}^{n}-\rho_{i}^{n} \frac{J_{i}^{n+1}-J_{i}^{n}}{J_{i}^{n+1}}=\rho_{i}^{n}\left(1-\frac{J_{i}^{n+1}-J_{i}^{n}}{J_{i}^{n+1}}\right)=\rho_{i}^{n} \frac{J_{i}^{n}}{J_{i}^{n+1}}
$$

or $\rho_{i}^{n+1} J_{i}^{n+l}=\rho_{i}^{n} J_{i}^{n}$, which is the Lagrange statement of conservation of mass (i.e., $\rho J=$ constant in time). This relation dictates the time evaluation of the denominator of $\mathrm{J}_{\mathrm{t}} / \mathrm{J}$ as $\mathrm{J}^{\mathrm{n}+1}$ instead of a centered value $\mathrm{J}^{\mathrm{n}+1 / 2}$.

This situation is common to each of the equations (27) through (30) and we wish a difference interpretation of the Jacobian $\mathrm{J}^{\mathrm{n}}$.

In the following theorem we find the Jacobian interpreted as the area ${ }^{6}$ of the boundary zone.

THEOREM 5: If a region $r$ in $(a, b)$-space maps onto a region $R$ in $(x, y)-$ space by the transformation $x=x(a, b, t), \bar{y}=y(a, b, t)$, and if the Jacobian of the transformation $J(x, y)=\left(\frac{x, y}{a, b}\right)$ exists and is positive everywhere in $R$, then there exists a point $(\xi, \eta)$ in $\mathrm{R}$ such that 


$$
J(\xi, \eta)=\frac{\iint_{R} d x d y}{\iint_{r} d a d b}
$$

PROOF: : By hypothesis $|\mathrm{J}|=\mathrm{J}$, and therefore we have $\iint_{\mathrm{R}} \mathrm{dxdy}=\iint_{\mathrm{r}} \mathrm{J}$ dadb; and from the mean value theorem there exists a point $(\xi, \eta)$ in $R$ such that $J(\xi, \eta) \iint_{r} \mathrm{dadb}=\iint_{\mathrm{r}} \mathrm{Jdab}$, from which (34) follows.

COROLLARY: For the equations of hydrodynamics the Lagrange variables $(a, b)$ can always be chosen so that $\mathrm{J}(\mathrm{x}, \mathrm{y})>0$.

PROOF: We established the corollary by choosing the Lagrange variable a and $b$ to be the initial positions of the fluid elements $(x(a, b, 0)=a$ and $y(a, b, 0)=b)$. Then $J^{\circ}(x, y)=J(x(a, b, 0), y(a, b, 0))=J(a, b)=1$ and from the conservation of mass we have $\rho J=\rho^{0} J^{0}=\rho^{0}$. where $\rho^{\circ}$ is the initial density $\dot{Q}(a, b, 0)$. Now, since density is always positive we must have $J(x, y)>0$.

We see from (33) that the difference equations deal only with the ratin of Jacobians; hence the underlying $(a, b)$ space and the initial value $\left(\mathrm{J}^{\circ}\right)$ of the Jacobian can be choosen arbitrarily. In the difference equations we shall identify the Jacobian $\mathrm{J}^{\mathrm{n}}$ with the area of the boundary zone at the nth time step. For the boundary zone calculations certain sequenres must be : determined and these are defined in the next section.

\section{BOUNDARY ZONES AND BOUNDARY. SEQUENCES}

The boundary zones are formed by the intersection of a polygonal line Lagrange boundary (that is, we assume the boundary is defined by a polygonal line which moves with the fluid) and the fixed Eulerian grid. Most often the resulting boundary zones will be a simply connected polygon whose sides are formed by part of the original fixed grid and portions of the polygonal Lagrarige 
line. It is entirely possible that the Lagrange boundary will decompose an Eulerian cell into several disjoint pieces and one must provide for this possibility in the difference equations.

To make the description of a boundary zone precise we define the following terms.

We suppose the polygonal Lagrange line parameterized with parameter $s(0<s<1)$ such that for increasing values of $s$ the Lagrange line is traversed with the Eulerian grid to the left. Denote the Lagrange line at the nth time step by

$$
L^{n}(s)=\left[<x^{n}(s), y^{n}(s)>\mid 0<s<1\right]
$$

Consider the point set A consisting of (a) the vertices of $L^{n}(s)$ and $(b)$ the intersections of $L^{n}(s)$ with the grid lines of the Eulerian mesh. Let the members of $A$ be arranged in the order of increasing $s$, and call this sequence $\left\{\mathrm{L}_{\mathrm{i}}^{\mathrm{n}}\right\}$.

Since the Eulerian fluid region in the $(x, y)$ plane (with boundary $L_{:}^{n}(s)$ ) is taken to be on the left when traversing $L^{n}(s)$ in the direction of increasing $s$, this Eulerian fluid region (including the boundary $L^{n}(s)$ ) we denote by $E^{n}$.

Let $G_{k, l}$ be the grid point with coordinates $\left(x_{k}, y_{k}\right)$ and consider the rectangular cell of the mesh with corners $G_{k, \ell}, G_{k+1, \ell}, G_{k+1, \ell+1}, G_{k, \ell+1}$. Denote by $C^{n}(k, l)$ the part of this cell which is common with $E^{n} \cdot C^{n}(k, l)$ will be a boundary zone if part of its boundary, $\partial C^{n}(k, l)$, is contained in $L^{n}(s)$ (i. e., $\left.\partial C^{n}(k, l) \cap L^{n}(s) \neq 0\right)$. Since $L^{n}(s)$ is a polygonal line, boundary zones are composed of one or more polygonal regions, and for the difference equations we need the vertices (of the boundaries of these polygonal regions) ordered in a counterclockwise direction. These sequences (one for each polygon) are called boundary sequences and are denoted by

$$
\left\{V_{i}^{n}(k, l, j)\right\}{ }_{i=1,2, \ldots I_{j}^{n}, j=1,2, \ldots}
$$


where the index $j$ indicates the $j$ th polygon making up $\partial C^{n}(k, \ell) . \quad I_{j}^{n}-1$ is the number of vertices in the jth polygon and for the purposes of the line integral formulation we see that the first and last vertex must be the same point, i. e., $V_{l}^{n}(k, l, j)=V_{\cdot I_{j}^{n}}^{n}(k, l, j)$. We also denote the coordinates of these vertices by

$$
v_{i}^{n}(k, l, j)=\left(x_{i}^{n}(k, l, j), y_{i}^{n}(k, l, j)\right)
$$

The complete details of the construction of the boundary sequence (36) will not be pursued, but we note that the $V_{i}^{n}(k, l, j)$ are points of $\left\{L_{i}^{n}\right\}$ together with some of the lattice points $G_{k, \ell}, G_{k+1, \ell}, G_{k+1, \ell+1}, G_{k, \ell+1}$. These are easily determined by starting at the first $L_{i}^{n}$ intersecting $\partial C^{n}(k, l)$ and pro- . ceeding along $L^{n}(s)$ in a counterclockwise direction (i. e., in the direction of increasing s) until $L^{n}(s)$ leaves $C^{n}(k, l)$ and then continuing along $\partial C^{n}(k, l)$ in a counterclockwise direction. 'I'he boundary sequence then includes all of the grid points and $L_{i}^{n}$ that are met until the starting $L_{i}^{n}$ is reached. These $L_{i}^{n}$ and grid points make up the first $(j=1)$. boundary sequence and the process is repeated for the next (if any) $L_{i}^{n}$ in common with $\partial C^{12}(k, l)$. For particular examples see Figs. 6 and 7.

For the divergence expressions in the boundary difference equations it is necessary to determine the relative velocity, $\underset{\sim}{\Omega}=\omega \underset{\sim}{i}+\sigma \underset{\sim}{j}$ at each of the vertices $V_{i}^{n}(k, l, j)$ or the average value of $\underset{\sim}{\Omega}$ between $V_{i}^{n}(k, l, j)$ and $V_{i+1}^{n}(k, l, j)$. If we denote this average velocity by $\Omega_{1+1 / 2}^{n+1 / 2}(k, l, j)$ then.we will call the sequences of the components $\omega, \sigma$ of $\Omega_{i+1 / 2}^{n+l}(k, l, j)$ boundary velocity sequences or simply velocity sequences and denote them by

$$
\left\{\omega_{i+1 / 2}^{n+1 / 2}(k, l, j)\right\}_{i=0,2 \ldots I_{j}^{n}-1}\left\{\sigma_{i+1 / 2}^{n+l / 2}(k ; \ell, j)\right\}_{i=0,1 \ldots I_{j}^{n}-1}
$$


As noted previously, if the line segment joining $V_{i}^{n}(k, l, j)$ and $V_{i+1}^{n}(k, l, j)$ is part of the Lagrange line, $L^{n}(s)$, then $\dot{\omega}_{i+l / 2}^{n+l / 2}(\dot{k}, l, j)=\sigma_{i+1 / 2}^{n+l / 2}(k, l, j)=0$; if the line segment is part of the Eulerian grid, then the relative velocities reduce to the fluid velocities, $i$. e., $\omega_{i+1 / 2}^{n+1 / 2}(k, l, j)=u_{i+1 / 2}^{n+1 / 2}(k, l, j)$ and $\sigma_{i+1 / 2}^{n+l / 2}(k, l, j)=v_{i+1 / 2}^{n+l / 2}(k, \ell, j)$.

So far in the discussion of boundary zones we have been considering cells $C^{n}(k, l)$ in which the variables $p, q, s$, and $P$ are defined, i. e., these variables are considered to have a constant value in the region $C^{n}(k, l)$. The components of momentum are defined as constant in cells of a staggered mesh defined by the intersection of grid lines $x_{k+1 / 2}=\frac{1}{2}\left(x_{k}+x_{k+1}\right), y_{\ell+1 / 2}=\frac{1}{2}\left(y_{\ell}+y_{\ell+1}\right)$. If we let the lattice points of this mesh be denoted by $G_{k+1 / 2, \ell+1 / 2}=\left(x_{k+1 / 2}\right.$, $\left.\mathrm{y}_{\ell+1 / 2}\right)$ and let $\mathrm{C}^{\mathrm{n}}(\mathrm{k}+1 / 2, \ell+1 / 2)$ be the common part of the rectangle with corners. $G_{k+1 / 2, \ell+1 / 2}, G_{k-1 / 2, \ell+1 / 2}, G_{k-1 / 2, \ell-1 / 2}, G_{k+1 / 2, \ell-1 / 2}$, and $E^{n}$, then $M$ and $N$ are defined (as constant) over the cells $C^{n}(k+1 / 2, \ell+1 / 2)$.

Consequently we have momentum boundary zones (i. e. those $C^{n}(k+1 / 2, \ell+1 / 2)$ which have part of their boundary $\partial C^{n}(k+1 / 2, l+1 / 2)$ in common with $\left.L^{n}(s)\right)$. and it is necessary to construct momentum boundary sequences which we denote by

$$
\left\{V_{i}^{n}(k+1 / 2, \ell+1 / 2, j)\right\}_{i=1,2 \ldots, I_{j}^{n}(k+1 / 2, \ell+1 / 2)}
$$

and boundary velocity sequences denoted by

$$
\begin{aligned}
& \left\{\omega_{i+1 / 2}^{n+1 / 2}(k+1 / 2, \ell+1 / 2)\right\} \\
& i=0,2, \ldots, I_{j}^{n}(k+1 / 2, \ell+1 / 2)-1 \\
& \left\{\begin{array}{c}
n+1 / 2 \\
i+1 / 2
\end{array}(k+1 / 2, \ell+1 / 2)\right\} \\
& \left.i=0,2, \ldots, I_{j}^{n}(k+1 / 2, \ell ! 1 / 2)-1\right)
\end{aligned}
$$


where these sequences have the same definition relative to the momentum cells $C^{n}(k+1 / 2, \ell+1 / 2)$ as $(37)$ and (38) had relative to the cells $C^{n}(k, \ell)$.

In addition to (39) and (40) the momentum equations involve the pressure gradient and thus it is necessary to construct a pressure (plus artificial viscosity $\bar{P}=P+q$ ) sequence denoted by

$$
\left\{\bar{P}_{i}^{n}(k+1 / 2, \ell+1 / 2)\right\}_{i=1,2, \ldots, I_{j}^{n}(k+1 / 2, \ell+1 / 2)}
$$

where the $\overline{\mathrm{P}}_{\mathrm{i}}^{\mathrm{n}}(\mathrm{k}+1 / 2, \ell+1 / 2)$ are the pressure and artificial viscosity in $\mathrm{E}^{\mathrm{n}}$ at the points $V_{i}^{n}(k+1 / 2, \ell+1 / 2) .7$

IX. BOUNDARY ZONE DIFFERENCE EQUATIONS FOR THE VARIABLE $\rho, \epsilon$, ETC. WHICH ARE CENTERED IN THE BOUNDARY ZONES $C^{n}(k, l)$.

We shall first consider the boundary zone difference equations. for the variables $\rho, q, \epsilon$ and $P$ which are centered in the cells $C^{n}(k, l)$. (That is, these variables are considered to have a constant value at each time step over the cells $\left.C^{n}(k, \ell).\right)$

We consider that the boundary sequences (36) and (38) have been determined and we wish to define the difference approximations of the Jacobian $\mathrm{J}$ : (which from (34) is the area of $\mathrm{C}^{\mathrm{n}}(\mathrm{k}, \mathrm{l})$ ) and the divergence of any step function which is constant over $C^{11}(k, l)$ times the relative velocity $\underset{\sim}{\Omega}$ (i. e., $\underset{\sim}{\nabla}: f \Omega$ ): Then $\underset{\sim}{\nabla} \cdot \underset{\sim}{\mathrm{U}}$ is obtained from the fundamental identity (24).

We consider that we are dealing with a particular boundary cell $C^{\mathfrak{n}}(k, l)$ which is composed of $j^{n}(k, l)$ polygonal regions, and we shall understand that the variables $V ; x, y, w, \sigma$, etc., refer to $C^{n_{1}}(k, l)$ (i. e., for $x_{i}^{n}(k, l, j)$ we shall write $x_{i}^{n}(j)$, etc.). Accordingly, then

$$
j_{k+1 / 2, \ell+1 / 2}^{n}=\frac{1}{2} \sum_{j=1}^{j^{n}(k, l)} \sum_{i=1}^{T^{n}(j)}\left(x_{i+1}^{n}(j)+x_{i}^{n}(j)\right)\left(y_{i+1}^{n}(j)-y_{i}^{n}(j)\right) .
$$


where the subscript $k+1 / 2, \ell+1 / 2$ is used to be consistent with the notation used for the interior equations; (i. e., we are treating the dependent variables which for nonboundary zones are centered in space at the midpoints $k+1 / 2$, $\ell+1 / 2$ of the Eulerian cells).

In order to approximate $\underset{\nabla}{\nabla} \underset{\sim}{\Omega}$ we see from Eq. (l1) that it will be composed of a sum of terms of the form $(f \omega)_{i+1 / 2}\left(y_{i+1}-y_{i}\right)-(f \sigma)_{i+1 / 2}\left(x_{i+1}-x_{i}\right)$. Now $\omega_{i+1 / 2}$ and $\sigma_{i+1 / 2}$ have been defined, so we must determine $f_{i+1 / 2}$. As pointed out previously, the relative velocities $\omega$ and. $\sigma$ vanish on the Lagrange line $L^{n}(s)$ and reduce to $u$ and $v$ for the other polygonal line segments making. up the boundary of $C^{n}(k, l)$. These line segments are composed of portions of the sides of the original rectangular Eulerian cell and cons equently are either horizontal or vertical line segments. Along the horizontal line segments $y_{i+1}-y_{i}=0$ and along vertical segments $x_{i+1}-x_{i}=0$. Thus $f_{i+1 / 2}$ need only be defined for those cases where $\omega \Delta y \not 0$ and $\sigma \Delta x \neq 0$. There are four possibilities.for each of these nonvanishing terms.

$$
\text { Let } \omega_{i+1 / 2}\left(y_{i+1}-y_{i}\right)=u_{i+1 / 2}\left(y_{i+1}-y_{i}\right) \neq 0 \text { then } x_{i}=x_{k} \text { or } x_{i}=x_{k+1} \text {. }
$$

Letting $f_{k, l}^{n}$ be the value of the function $f$ over the cell $C^{n}(k, l)$ we define

$$
(f \omega)_{i+1 / 2}^{n+l / 2}(j)=\left\{\begin{array}{cc}
f_{k-1, l}^{n} & \text { if } x_{i}=x_{k} \text { and } u_{i+1 / 2}^{n+1 / 2}>0 \\
f_{k, l}^{n} & \text { if } x_{i}=x_{k} \text { and } u_{i+1 / 2}^{n+1 / 2}<0 \\
f_{k, l}^{n} & \text { if } x_{i}=x_{k+1} \text { and } u_{i+1 / 2}^{n+1 / 2}>0 \\
f_{k+1, l}^{n} & \text { if } x_{i}=x_{k+1} \text { and } u_{i+1 / 2}^{n+1 / 2}<0
\end{array}\right\}\left\{\begin{array}{l}
n+1 / 2(j) \\
u_{i+1 / 2}
\end{array}\right\}
$$




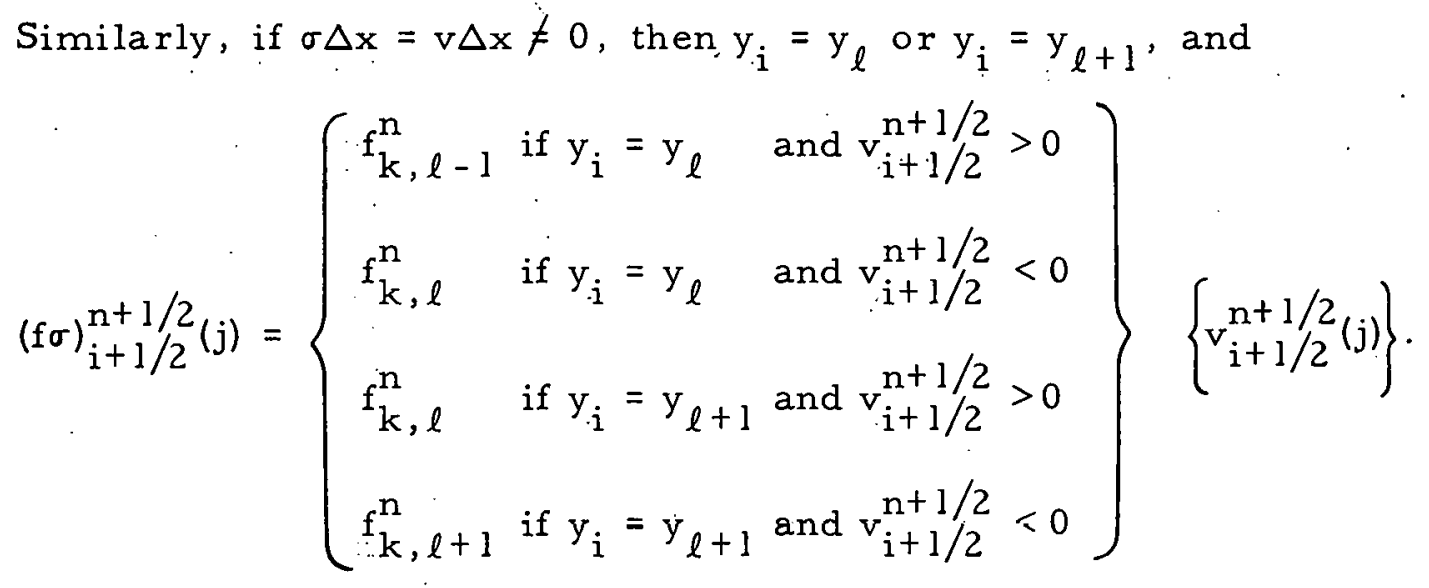

In terms of (42), (4:3) and (44) we define the divergence and (as in the case of the Jacobian) we us e the subscripts $k+1 / 2$ and $\ell+1 / 2$.

$$
\begin{aligned}
& \mathrm{n}+\mathrm{l} \text { n } \\
& {[\underset{\sim}{\nabla} \cdot \underset{\sim}{\sim}]_{k+1 / 2, \ell+1 / 2}^{n+1 / 2}=\frac{1}{J_{k+1 / 2, \ell+1 / 2}^{n+1}} \sum_{j=1}^{j(k, l)} \sum_{i=1}^{I(j)}\left\{(f \omega)_{i+1 / 2}^{n+1 / 2}(j)\left[y_{i+1}^{n+1 / 2}(j)-y_{i}^{n+1 / 2}(j)\right]\right.} \\
& \left.-(f \sigma)_{i+1 / 2}^{n+1 / 2}(j)\left[x_{i+1}^{n+1 / 2}(j)-x_{i}^{n+1 / 2}(j)\right]\right\} .
\end{aligned}
$$

Letting $\mathrm{f} \equiv \mathrm{l}$ and using $(24)$ we define

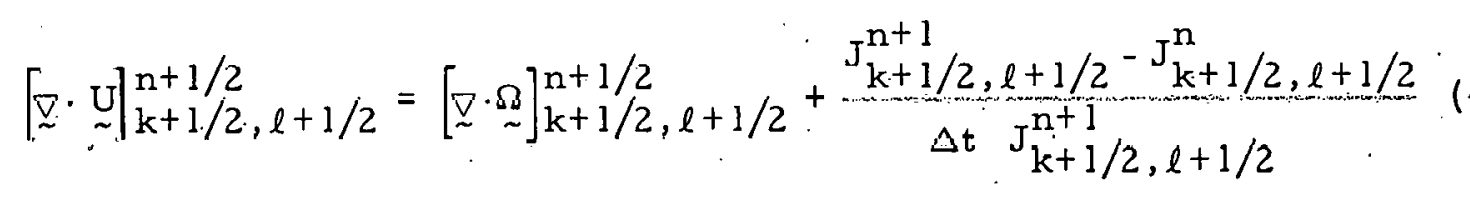

We can now give the difference approximation for equations (29), (30) and for $\dot{q}$ (q for the boundary zones is still given by (15)). Keeping in mind the discussion of (32) we have

$$
\rho_{k+1 / 2, \ell+1 / 2}^{n+1}=\rho_{k+1 / 2, \ell+1 / 2}^{n} \frac{J_{n+1 / 2, \ell+1 / 2}^{n}}{J_{k+1 / 2, \ell+1 / 2}^{n+1}} \cdot \Delta t[\bar{z} \cdot \rho \Omega]=\frac{n+1 / 2}{k+1 / 2, \ell+1 / 2}
$$




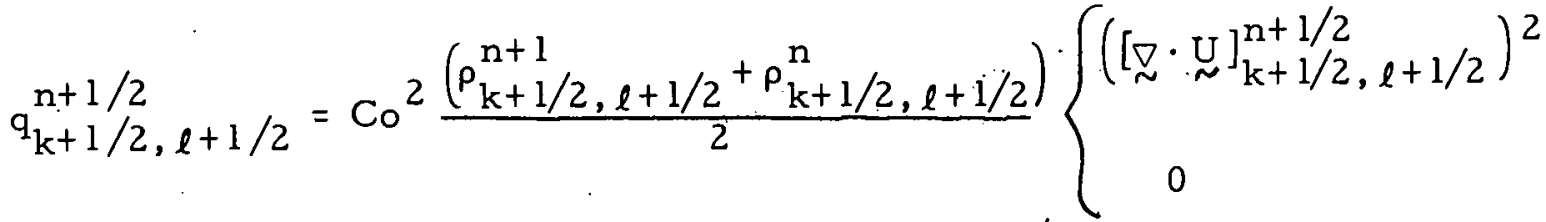

$$
\begin{aligned}
& \text { if }[\underset{\sim}{\nabla} \cdot \underset{\sim}{U}]_{k+1 / 2, \ell+1 / 2}^{n+1 / 2}<0 \\
& \text { if }[\underset{\sim}{\nabla} \cdot \underset{\sim}{U}]_{k+1 / 2, \ell+1 / 2}^{n+1 / 2} \geq 0
\end{aligned}
$$

$$
\begin{aligned}
\epsilon_{k+1 / 2, \ell+1 / 2}^{n+1}=\epsilon_{k+1 / 2, \ell+1 / 2}^{n} & \frac{J_{k+1 / 2, \ell+1 / 2}^{n}}{J_{k+1 / 2, \ell+1 / 2}^{n+1}}-\Delta t\left\{[\bar{\nabla} \cdot \epsilon \Omega]_{k+1 / 2, \ell+1 / 2}^{n+1 / 2}\right. \\
& \left.\left.+\bar{P}_{k+1 / 2, \ell+1 / 2}^{n+1 / 2} \underset{\sim}{\nabla} \cdot \underset{\sim}{\mathrm{U}}\right]_{k+1 / 2, \ell+1 / 2}^{n+1 / 2}\right\}
\end{aligned}
$$

where $\bar{P}=P+q$ and $P^{n+1 / 2}$ is obtained by iteration using $P^{n}$ as a first guess.

$$
P_{k+1 / 2, \ell+1 / 2}^{n+1}=P\left(\rho_{k+1 / 2, \ell+1 / 2}^{n+1}, \epsilon_{k+1 / 2, \ell+1 / 2}^{n+1}\right) .
$$

A special situation develops when the area, $J_{k+1 / 2, l+1 / 2}^{n}$ of $C^{n}(k, l)$ becomes some small fraction of the area of the original Eulerian cell. If the boundary zone calculation were to proceed as $\mathrm{J}_{\mathrm{k}+1 / 2, \ell+1 / 2}^{\mathrm{n}} \rightarrow 0$ then the time interval for stability would also approach zero. To avoid this, the boundary zone $C^{n}(k, l)$ can be blended with an adjacent zone, i. e., $C^{n}(k, l)$ and one of its neighbors are combined to form a single new boundary zone. This technique we all blending, and it only slightly perturbs the fluid flow. A somewhat cruder possibility is to declare $C^{n}(k, l)$ empty when $J_{k+1 / 2, \ell+1 / 2}^{n}$ is small and redistribute its mass, internal energy, and the PdV work (in $\mathrm{J}_{\mathrm{k}+1 / 2, \ell+1 / 2}^{\mathrm{n}}$ going to zero) to an adjacent zone. This technique has been used quite successfully in CEL and leads to a fluctuation in the flow of a few percent. 
X. DIFFERENCE EQUATIONS FOR THE COMPONENTS OF MOMENTUM ( $m=\rho u, n=\rho v$ ) WHICH ARE CENTERED IN THE BOUNDARY ZONES $C^{n}(k+1 / 2, \ell+1 / 2)$

As in the preceding discussion, we must define the Jacobian, divergence and in addition we must appeal to (9) and (10) for the approximation of the pressure gradients over the momentum boundary zones $C^{n}(k+1 / 2, \ell+1 / 2)$. Since the Jacobian and divergence have precisely the same definition relative to the momentum mesh as they did for the fundamental mesh (composed of the $C^{n}(k, l)$ ) we simply state

$J_{k, \ell}^{n}=\frac{1}{2} \sum_{j=1}^{j^{n}(k+1 / 2, \ell+1 / 2)} \sum_{i=1}^{I^{n}(j)}\left(x_{i+1}^{n}(j)+x_{i}^{n}(j)\right)\left(y_{i+1}^{n}(j)-y_{i}^{n}(j)\right)$

and for any $f$ defined over $C^{n}(k+1 / 2, \ell+1 / 2)$

$$
\begin{aligned}
{[\bar{\nabla} \cdot f \underset{\sim}{\Omega}]_{k, \ell}^{n-1 / 2}=\frac{1}{J_{k, \ell}^{n}} \sum_{i=1}^{j} \sum_{i=1}^{n}[(k, l)} & {\left[(j)_{i+1 / 2}^{n-1 / 2}\left(y_{i+1}^{n-1 / 2}(j)-y_{i}^{n-1 / 2}(j)\right)\right.} \\
& \left.-(f \sigma)_{i+1 / 2}^{n-1 / 2}\left(x_{i+1}^{n-1 / 2}(j)-x_{i}^{n} 1 / 2(j)\right)\right] .
\end{aligned}
$$

where $f_{i+1 / 2}^{n-1 / 2}$ has the same definition relative to $C^{n}(k+1 / 2, \ell+1 / 2)$ as $f_{i+1 / 2}^{n}$ had to $C^{n}(k, l)$ in $(43)$ and $(44)$.

The components of the pressure gradient are approximated using (9), (10), (42) and taking an area weighted average of the derivatives for each of the $j^{n}(k+1 / 2, l+1 / 2)$ polygonal regions of $C^{n}(k+1 / 2, i+1 / 2)$. We note that. the denominator of (9) and (10) is twice the area of the polygonal region under consideration and that the Jacobian, $\mathrm{J}_{\mathrm{k}, \ell}^{\mathrm{n}}$, is the sum of the areas of the se polygons: Hence

$$
\left(\frac{\Delta \bar{P}}{\Delta x}\right)_{k, l}^{n}=\frac{1}{2 J_{k, \ell}^{n}}
$$$$
\sum_{j=1}^{n}(k+1 / 2, \ell+1 / 2)
$$$$
\sum_{i=1}^{I^{n}(j)}\left(\bar{P}_{i}^{n}(j)+\bar{P}_{i+1}^{n}(j)\right)\left(y_{i+1}^{n}(j)-y_{i}^{n}(j)\right)
$$ 
and

$$
\left(\frac{\Delta \bar{P}}{\Delta y}\right)_{k, \ell}^{n}=\frac{-1}{2 J_{k, \ell}^{n}} \sum_{j=1}^{j^{n}(k+1 / 2, \ell+1 / 2)} \sum_{i=1}^{I^{n}(j)}\left(\bar{P}_{i}^{n}(j)+\bar{P}_{i+1}^{n}(j)\right)\left(x_{i+1}^{n}(j)-x_{i}^{n}(j)\right) \text {. }
$$

We are now able to approximate the momentum equations (27) and (28) for the boundary zones $C^{n}(k+1 / 2, \ell+1 / 2)$.

$$
\begin{aligned}
& M_{k, \ell}^{n+1 / 2}=M_{k, \ell}^{n-1 / 2}\left(J_{k, \ell}^{n-1} / J_{k, \ell}^{n}\right)-\Delta t\left\{[\underset{\sim}{\nabla} \cdot \underset{\sim}{\Omega}]_{k, \ell}^{n-1 / 2}+\left(\frac{\Delta \bar{P}}{\Delta x}\right)_{k, \ell}^{n}\right\} \\
& N_{k, \ell}^{n+l / 2}=N_{k, \ell}^{n-1 / 2}\left(J_{k, \ell}^{n-1} / J_{k, \ell}^{n}\right)-\Delta t\left\{[\underset{\sim}{\nabla} \cdot \underset{k, \ell}{N}]_{k, l}^{n-1 / 2}+\left(\frac{\Delta \bar{P}}{\Delta x}\right)_{k, \ell}^{n}\right\} .
\end{aligned}
$$

In order to define the velocity we need an average density for $C^{n}(k+1 / 2 ; \ell+1 / 2)$. This is defined as the area weighted average of the neighboring densities. Thus

$$
\rho_{k, l}^{n+1 / 2}=\frac{\sum_{i}(\rho J)_{i}^{n}}{\sum_{i} j_{i}^{n}}
$$

where $(\rho J)_{i}^{n}$ is the mass in the ith (nonempty) $C^{n}(k, l)$ adjacent to $C^{n}(k+1 / 2$, $\ell+1 / 2)$. Then

$$
\left.\begin{array}{l}
u_{k, \ell}^{n+1 / 2}=\frac{M_{k, \ell}^{n+1 / 2}}{\rho_{k, \ell}^{n}} \\
v_{k, \ell}^{n+l / 2}=\frac{N_{k, \ell}^{n+1 / 2}}{\rho_{k, \ell}^{n}}
\end{array}\right\} \text {. }
$$

A special situation develops when the grid point $\left(\mathrm{x}_{\mathrm{k}, \ell}, \mathrm{y}_{\mathrm{k}, \ell}\right)$ is covered by the Lagrange region, i.e., $\left(x_{k, \ell}, y_{k, \ell}\right)$ is not in $E^{n}$. Then (55) and (56) are used to determine the tangential components of momentum and the normal components are constructed from the motion of the Lagrange boundary $L^{n}(s)$. 
This completes the discussion of the Eulerian calculation and next we briefly take up the Lagrange calculations.

The situation here is quite straightforward as one need specify only the pressure at the Lagrange Eulerian interface $L^{n}(s)$ to determine the Lagrange calculation completely. In CEL, this boundary pressure is taken to be the area-weighted average of the pressures in all the Eulerian boundary zones which have part of their boundary in common with a given straight line segment of $\mathrm{L}^{\mathrm{n}}(\mathrm{s})$. (See Fig. 8.) This boundary prèssure, although not quite correct, ${ }^{8}$ is a satisfactory choice in practice. We proceed then to the Lagrange calculations.

\section{LAGRANGE DIFFERENCE EQUATIONS}

The following equations provide a simple and accurate set of Lagrange difference equations. Our approximating mesh is quadrilateral.in terms of the space variables $x=x(a, b, t), y=y(a, b, t)$ (where $a$ and $b$ are the Lagrange coordinates); and the space and time centering of $u, v, p$, etc. are the same as in the Eulerian equations. We will again use the approximations of Theorem 1 and Theorem 5 and thus we have an over-all consistency to the difference equations in CEL. It is also interesting to note that the resulting Lagrange difference equations are identical to the usual differenclng where the partial derivatives with respect to $x$ and $y$ are expressed in terms of the partial derivatives with respect to the Lagrange variables $a$ and $b$ and these derivatives are then approximated on a square mesh in the $(a, b)$ space. (See Fig. 3.) This again shows the "naturalness" of the approximations given by Theorem 1. The use of the se approximations is also satisfactory in that no reference is made to the underlying $(a, b)$-space. Consequently, one could just as easily approximate the Lagrange equations over an arbitrary (instead of a quadrilateral) mesh, in $(x, y)$-space. 
The differential equations in Lagrange coordinates are given by:

$$
\begin{aligned}
& u_{t}=-\frac{1}{\rho} P_{x} \\
& v_{t}=-\frac{1}{\rho} P_{y} \\
& x_{t}=u \\
& y_{t}=v \\
& \rho J=\text { Constant } \\
& e_{t}=\frac{P}{\rho} \rho_{t} \\
& P=P(e, \rho)
\end{aligned}
$$

Where $J$ is the Jacobian $\left(\frac{x, y}{a, b}\right)$ and e here is the internal energy per unit mass.

The following definition uses the numbering in Fig. 9 and is based on equations (9), (10), (11) and Theorem 5 (which approximated the Jacobian as an area).

$$
\mathrm{J}_{\mathrm{k}+1 / 2, \ell+1 / 2}^{\mathrm{n}}=\frac{1}{2}\left\{\left(\mathrm{x}_{3}-\mathrm{x}_{1}\right)\left(\mathrm{y}_{4}-\mathrm{y}_{2}\right)-\left(\mathrm{x}_{4}-\mathrm{x}_{2}\right)\left(\mathrm{y}_{3}-\mathrm{y}_{1}\right)\right\}^{\mathrm{n}}
$$

and letting $\overline{\mathrm{P}}=\mathrm{P}+\mathrm{q}$

$$
\begin{aligned}
& \text { (ii) }[\overline{\mathrm{P}}, \mathrm{x}]_{\mathrm{k}, \ell}^{\mathrm{n}}=\frac{1}{8}\left\{\left(\overline{\mathrm{P}}_{11}-\mathrm{P}_{13}\right)\left(\mathrm{x}_{4}+\mathrm{x}_{5}+\mathrm{x}_{6}-\mathrm{x}_{8}-\mathrm{x}_{9}-\mathrm{x}_{2}\right)\right. \\
& \left.-\left(\bar{P}_{12}-\bar{P}_{10}\right)\left(x_{2}+x_{3}+x_{4}-x_{6}-x_{7}-x_{8}\right)\right\}^{n} \\
& (\rho J)_{k, \ell}^{\mathrm{n}}=\frac{1}{4}\left\{(\rho J)_{10}^{\mathrm{n}}+(\rho J)_{11}^{\mathrm{n}}+(\rho J)_{12}^{\mathrm{n}}+(\rho J)_{13}^{\mathrm{n}}\right\} \\
& \text { (iv) }\left[u_{x}+v_{y}\right]_{k+1 / 2, \ell+1 / 2}^{n+1 / 2}=\frac{1}{J_{k+1 / 2, \ell+1 / 2}^{n+1}}\left\{\sum _ { i = 1 } ^ { 3 } \left[\left(\frac{u_{i+1}+u_{i}}{2}\right)^{n+1 / 2}\left(y_{i+1}-y_{i}\right)^{n+1 / 2}\right.\right. \\
& \left.\left.-\left(\frac{v_{i+1}+v_{i}}{2}\right)^{n+1 / 2}\left(x_{i+1}-x_{i}\right)^{n+1}\right]\right\}
\end{aligned}
$$


The Lagrange difference equations are then given by:

$$
\begin{aligned}
& u_{k, l}^{n+1 / 2}=u_{k, l}^{n-1 / 2}-\Delta t \frac{[\bar{P}, y]_{k, \ell}^{n}}{(\rho J)_{k, \ell}^{n} \vdots} \\
& v_{k, l}^{n+1 / 2}=v_{k, l}^{n-1 / 2}+\Delta t \frac{[\bar{P}, x]_{k, \ell}^{n}}{(p J)_{k, \ell}^{n}} \\
& \left.\begin{array}{l}
x_{k, \ell}^{n+1}=x_{k, \ell}^{n}+\Delta t u_{k, \ell}^{n+1 / 2} \\
y_{k, \ell}^{n+1}=y_{k, \ell}^{n}+\Delta t v_{k, \ell}^{n+1 / 2}
\end{array}\right\} \\
& \rho_{k+1 / 2, \ell+1 / 2}^{n+1}=\rho_{k+1 / 2, \ell+1 / 2}^{n} \frac{J_{k+1 / 2, \ell+1 / 2}^{n}}{J_{k+1 / 2, \ell+1 / 2}^{n+1}}
\end{aligned}
$$

$\mathrm{q}_{k+1 / 2, \ell+1 / 2}^{n+1 / 2}=\frac{1}{2} C^{2}\left[(\rho J)_{k+1 / 2, \ell+1 / 2}^{n+1}+(\rho J)_{k+1 / 2, \ell+1 / 2}^{n}\left\{\begin{array}{c}{\left[\left(u_{x}+v_{y}\right)_{k+1 / 2, \ell+1 / 2}^{n+1 / 2}\right.} \\ 0\end{array}\right.\right.$

$$
\left.\begin{array}{l}
\text { if }\left(u_{x}+v_{y}\right)_{k+1 / 2, \ell+1 / 2}^{n+1 / 2}<0 \\
\text { if }\left(u_{x}+v_{y}\right)_{k+1 / 2, \ell+1 / 2}^{n+1 / 2} \geq 0
\end{array}\right\}
$$

$$
\begin{gathered}
e_{k+1 / 2, \ell+1 / 2}^{n+1}=e_{k+1 / 2, \ell+1 / 2}^{n}+\bar{P}_{k+1 / 2, \ell+1 / 2}^{n+1 / 2} \frac{\left(\rho^{n+1}-\rho^{n}\right)_{k+1 / 2, \ell+1 / 2}}{\left(\rho^{n+1} \rho^{n}\right)_{k+1 / 2, \ell+1 / 2}} \\
P_{k+1 / 2, \ell+1 / 2}^{n+1}=P\left(e_{k+1 / 2, \ell+1 / 2}^{n+1}, \rho_{k+1 / 2, \ell+1 / 2}^{n+1}\right)
\end{gathered}
$$

where $P_{k+1 / 2, \ell+1 / 2}^{n+1 / 2}$ in (65) is obtained by using $P_{k+1 / 2, \ell+1 / 2}^{n}$ as a first estimate in (65) and averaging the result of (66) with $P_{k+1 / 2, l+1 / 2}^{\mathrm{n}}$ for the final estimate.

The velocity equations (60) and (61) must be modified for the points of the lattice which define the boundaries of the Lagrange region, but the remaining equations hold for all points of the mesh. The modifications to (60) and 
(61) are straightforward and simply require a consistent application of (9) and (10) to determine $\mathrm{P}_{\mathrm{x}}$ and $\mathrm{P}_{\mathrm{y}}$ (where the pressure acting on these boundaries is determined from the Eulerian region or is a given boundary value). As an example, see Fig. 10.

This completes the description of a typical Lagrange region in CEL. It should be pointed out that as elaborate Lagrange calculations as desired can just as easily be coupled to the Eulerian region, and of course (as is the case of the CEL code) several independent Lagrange regions can be us ed to help represent more complicated geometries.

\section{DISCUSSION AND GRAPHICAL RESULTS OF} SEVERAL CEL CALCULATIONS

The following examples are intended to exhibit the fundamentals of the CEL code rather than to present the solution of particularly difficult or significant problems.

In order to display the fluid flow, it has been found most useful to plot the fluid velocity vectors at each point of the Eulerian mesh. For the Lagrange regions, we plot the position of the Lagrange grid points and the lines joining these grid points. The plotting is done automatically as part of the output using a cathode ray tube which is associated with the computer.

In the first problem (see Fig. . ll) we consider Mach $\infty$ :flow of a perfect gas $(\gamma=1.4)$ past a rigid disk. The initial conditions in the gas are zero temperatures (hence zero sound speed), constant density, and constant velocity parallel to the axis of revolution ( $Z$ axis). The gas is given an Eulerian representation and the rigid disk is represented by a simple stationary Lagrange grid (labeled $L_{1}$ in the figures).

Figure lla shows the gas flow shortly after start up. Cavitation occurs - behind the disk and the shocked gas begins to expand into this region. The 
gas is essentially brought to rest in front of the disk and a bow shock forms and begins to move out to the left.

Figures $1 \mathrm{lb}$ through 1 lf show subsequent times and illustrate the development of a circulating region behind the disk and the establishment of a stationary bow shock. Figure I If is essentially the steady-state configuration of the flow past the disk.

The second CEL problem is again Mach $\infty$ flow of a perfect gas past a dense (but compressible) metal sphere. Here a bow shock forms and a shuck is transmitted into the sphere.

In this problem the gas is again approximated by the Eulerian grid. However, in order to have an example of a problem with two Eulerian regions approximating different fluids, the sphere was deliberately decomposed into a central sphere and a outer spherical shell. The outer spherical shell is approximated by a Lagrange grid and the central sphere is approximated by the Eulerian mesh. This.is illustrated in Fig. 12a.

Figure $12 b$ is a plot of the problem shortly after $t=0$. The remaining figures (Figs. $12 \mathrm{c}$ through $12 \mathrm{~g}$ ) show the flow in the gas and the progress of the transmitted shock through the sphere. In the resulting deformation of the sphere we see (at the leading edge) the rapid growth of a spike and a bubble which is characteristic of Taylor unstable flow.

This type of instability arises when a less dense fluid accelerates a : more dense fluid las is the case of the gas accelerating the more dense sphere). The nature of the instability is such that any perturbation of the interface separating the two fluids will grow in time with the amplitudes of the shorter wavelength components of the perturbation growing the more rapidly. 
In this problem the start-up conditions introduced a slight dent in the first two Lagrange zones representing the leading edge of the sphere. This dent corresponds to a short wavelength; consequently a spike and bubble were formed.

This calculation points out one of the difficulties present in two space dimensional problems. Namely, if the flow is physically unstable, then any perturbation of the interface separating two fluids can quickly grow and may ultimately make the problem meaningless.

Since the flow may be perturbed (locally) as much as a few percent in the CEL calculations, (due to the "scavenging" - or uncovering; see Section IX) of fractional boundary zones, we can expect trouble in problems where the flow may, be unstable. The effect of such perturbations in small boundary zones can be minimized by using a "blending" calculation (s ee Section IX) instead of the cruder "scavenging" calculation and also by the use of a finer Eulerian mesh relative to the Lagrange mesh. By using a more refined Eulerian mesh the Lagrange boundary "sees" an integrated.pressure field and essentially any perturbations are smoothed out. Experience has shown that sufficient accuracy is obtained when the zones of the Eulerian mesh are roughly one third the area of the zones of the Lagrange mesh.

In CEL there is also a provision for the introduction of an artifical surface tension term ${ }^{9}$ which can act selectively on any Lagrange-Eulerian interface. Its effect is to damp out short wave length irregularities of the Lagrange interface (i.e., wavelengths represented by two or three Lagrange mesh lengths) but does not damp longer wavelengths. This surface tension term was not used in this second example nor was the Eulerian mesh size sufficiently refined for the size of the Lagrange mesh that was used. (See Fig. 12a.) 
In the third example (see Fig. 13a) we consider a region occupied by three different fluids (regions I, II and III, and IV). Initially we suppose that a plane shock has been established by a piston (region I) moving to the right and that at $t=0$ for our problem the shock front has just reached the dotted line separating regions II and III. Here regions II and III have the same equation of state but correspond to shocked gas in II and unshocked gas in III. Region IV is a thin but dense piston which is free to move when the resulting shock front reaches it.

The boundary conditions for this problem are: a constant pressure $P_{1}$ is maintained on the piston surface $D_{1}$ and a zero pressure on the free surface $\mathrm{D}_{4}$. The remaining boundaries of the problem are rigid walls (hatched regions) hence the component of velocity normal to these surfaces is zero.

If we let $\rho_{I}^{0}$ denote the initial density of region $I, \rho_{\text {II }}^{0}$ the initial density of region.II, etc., then the initial conditions are:

$$
\begin{aligned}
& p_{I}^{0}=p_{I I}^{0}=p_{I I I}^{0} \because p_{I X}^{0} ; \quad P_{I}=P_{I}^{0}=P_{I I}^{0}, P_{I I I}^{0}=P_{I V}^{0}=0 ; \\
& u_{I}^{0}=u_{I I}^{0}>0, u_{I I I}^{0}=u_{I V}^{0}=0 ; \text { and } v_{I}^{0}=v_{I I}^{0}=v_{I I I}^{0}=v_{I V}^{0}=0 .
\end{aligned}
$$

Figure $13 \mathrm{~b}$ shows the initial Eulerian and Lagrange grids. Figure $13 \mathrm{c}$ is shortly after $\mathrm{t}=0$ and we see that the shock is continuing into region III and that it is reflecting from the rigid wall in the upper half of region II.

In Fig. 13d we see that the high pressure behind the reflected shock has resulted in flow around the corner of the rigid wall and the shock proceeding into region III is no longer plane.

In Fig. 13e the reflected shock has reached the piston (region-I) and has begun to slow it down. In Fig. 13f, the reflected shock has turned the. upper half of region I around and it has begun to expand to the left. . The 
curved shock has also reached region IV and a shock proceeds into the upper half of this region.

In Fig. 13f the shock is through the upper half of region IV and the surface $\mathrm{D}_{4}$ is beginning to expand into a vacuum. In Fig. $13 \mathrm{~g}$ through $13 \mathrm{i}$ we continue to follow the flow. We see that the gas flow around the corner has reached the $\mathrm{x}$ axis and produces a reflection of the flow and a shock is produced which stops the forward motion of the lower half of region I. The problem was discontinued at this time.

\section{A.CKNOWLEDGMENTS}

I specifically wish to acknowledge the great assistance of Patrick W. Crowley in helping with the initial development of CEL and the subsequent development of the variety of routines (many of them quite novel - as in the case of the graphical display of the flow fields, etc.) which are necessary in order to transform a skeleton code into a useful production code. And a special acknowledgment is due Shirley M. Campbell for her absolutely amazing job of programming this most difficult code. The actual existence of CEL is certainly due to her, and thanks to her clear understanding of the entire problem, she was able to proceed entirely on her own and to offer many helpful suggestions. I further wish to thank Professor Murry H. Protter of the University of California at Berkeley for his many helpful suggestions in preparing this article. 


\section{REFERENCES}

von Neumann, J. and Richtmyer (1950), J. A.ppl. Phys. 21, 232. 


\section{FOOTNOTES}

* Work done under the auspices of the U. S. Atomic Energy Commission.

${ }^{1} U_{p}$ to 7000 mesh points are provided for in the IBM 7.094 version of CEL and up to 20,000 mesh points can be used in the IBM 7030 (Stretch) version of the CEL code.

${ }^{2}$ It is clear that if the functions are extended between mesh points by higher order (or other) interpolating functions then a different approximation of derivatives is obtained.

${ }^{3}$ Another possibility is $\frac{u_{1}+u_{2}}{2}$ for $\frac{u_{5}+u_{6}}{2}$.

4 Another possibility is $\frac{v_{2}+v_{3}}{2}$ for $\frac{v_{5}+v_{7}}{2}$.

${ }^{5}$ The fundamental identity corresponding to (24) for the axially symmetric case is $\underset{\sim}{\nabla} \cdot \underset{\sim}{U} \equiv \underset{\sim}{\nabla}+\frac{(\mathrm{RJ})}{(\mathrm{RJ})}$ where $\mathrm{R}=\left(\mathrm{x}^{2}+\mathrm{y}^{2}\right)^{1 / 2}$.

${ }^{6}$ For the axially symmetric case (where $R=:\left(x^{2}+y^{2}\right)^{1 / 2}$ ) the term $R J$ replaces $\mathrm{J}$ and $\mathrm{RJ}$ is likewise interpreted as the volume of revolution of the boundary zone.

${ }^{7}$ Actually, for those $V_{i}^{n}(k+1 / 2, \ell+1 / 2)$ which are vertices $L_{i}^{n}$ of the Lagrange line $L^{n}(s)$, we should use a boundary pressure consistent with the fact that. pressure is continuous across a contact discontinuity, but in practice this is difficult to determine and using the Eulerian pressure has proven to be satisfactory.

${ }^{8} \mathrm{~L}^{\mathrm{n}}(\mathrm{s})$ is in general a contact discontinuity across which the pressure and the normal component of velocity are continuous. This implies that the pressure acting on $\mathrm{L}^{\mathrm{n}}(\mathrm{s})$ is a mass-weighted average of the Eulerian and Lagrange pressures.

${ }^{9}$ Robert Lelevier (formerly at LRL Livermore) first suggested and showed the usefulness of introducing an artificial surface tension term (in the same spirit as the use of an artificial viscosity) in order to study Taylor unstable flow. 


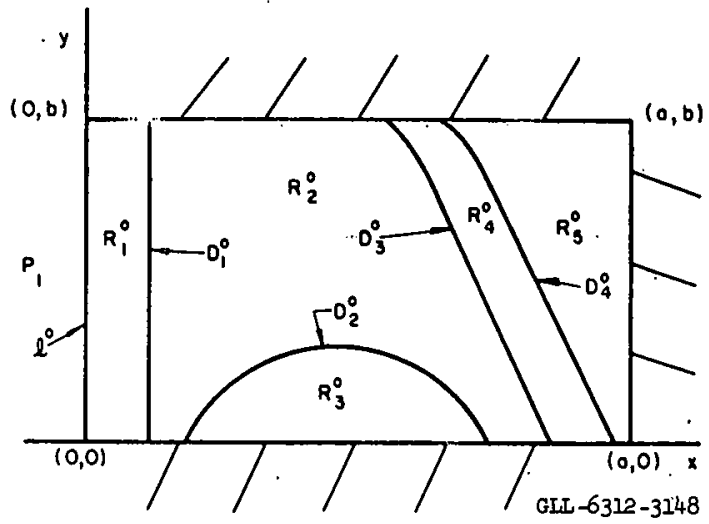

Fig. la. A simple multifluid problem with the initial $(t=0)$ conditions.

$$
\begin{aligned}
& \left.\mathrm{R}^{0}=\langle\mathrm{x}, \mathrm{y}\rangle \mid 0 \leq \mathrm{x} \leq \mathrm{a} \wedge 0 \leq \mathrm{y} \leq \mathrm{b}\right\} \\
& \ell^{0}=;\langle\mathrm{x}, \mathrm{y}\rangle \mid \mathrm{x}=0 \quad 0 \leq \mathrm{y} \leq \mathrm{b} ;
\end{aligned}
$$

Initial densities for regions $R_{1}^{0} \ldots R_{5}^{0}$ are $\rho_{1}^{0} \ldots \ldots \rho_{5}^{0}$, initial velocities are zero, and initial pressures are $P_{1}^{0}=P_{2}^{0}=P_{3}^{0}=P_{4}^{0}=P_{5}^{0}=P_{0}$.

The prescribed boundary conditions for $t \geq 0$ are $P_{l} \equiv$ const. on $l$ and $P_{1}>P_{0}$. The remaining boundary of $R$ is a rigid wall. This is, $u$ is the $x$ component of velocity, $v$ is the $y$ component of velocity, and $u(a, y, t)=v(x, 0, t)$ $=v(x, b, t)=0$.

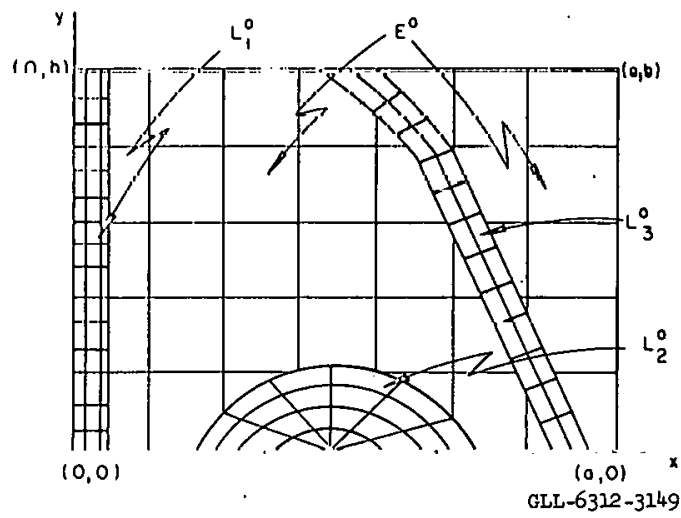

Fig. lb. A possible initial approximation of the problem in Fig. I using the CEL code.

$$
\begin{aligned}
& R^{0}=R(x, y, 0)=\{\langle x, y>| 0 \leq x \leq a \quad 0 \leq y \leq b\} \\
& \text { and we choose } R=R^{0} .
\end{aligned}
$$

$\mathrm{L}_{1}^{0}$ approximates $\mathrm{R}_{\mathrm{l}}^{0}$;

$\mathrm{L}_{2}^{0}$ approximates $\mathrm{R}_{3}^{0}$;

$\mathrm{L}_{3}^{0}$ approximates $\mathrm{R}_{4}^{0}$;

$E$ approximates $R=R^{0}$;

$E^{0}$ approximates $R_{2}^{0} \cup R_{5}^{0}$. 


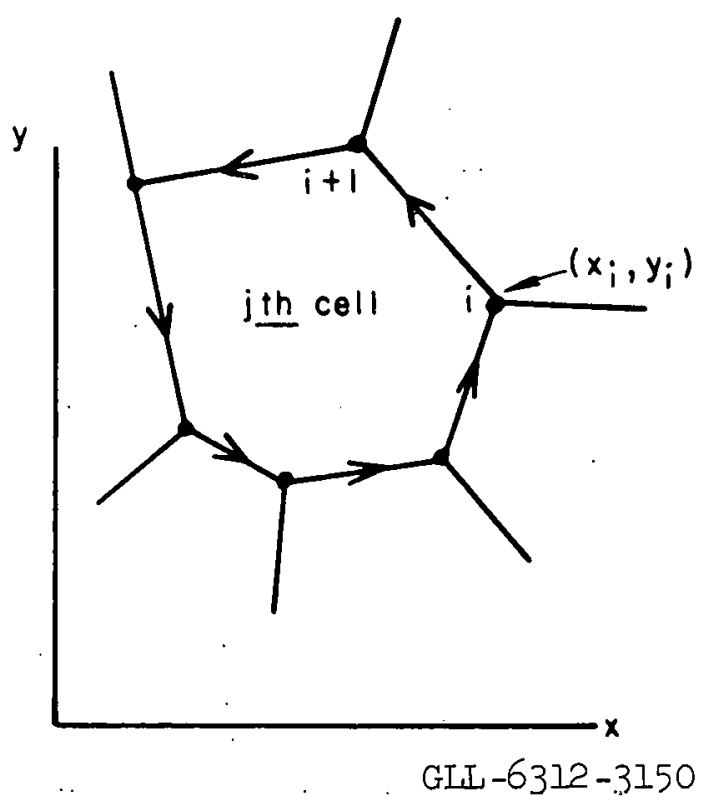

fig. 2. The jth cell of a general polygonal covering mesh. The $\mathrm{N}$ verices are numbered in a counter-clockwise direction $\left(x_{i}, y_{i}\right)$
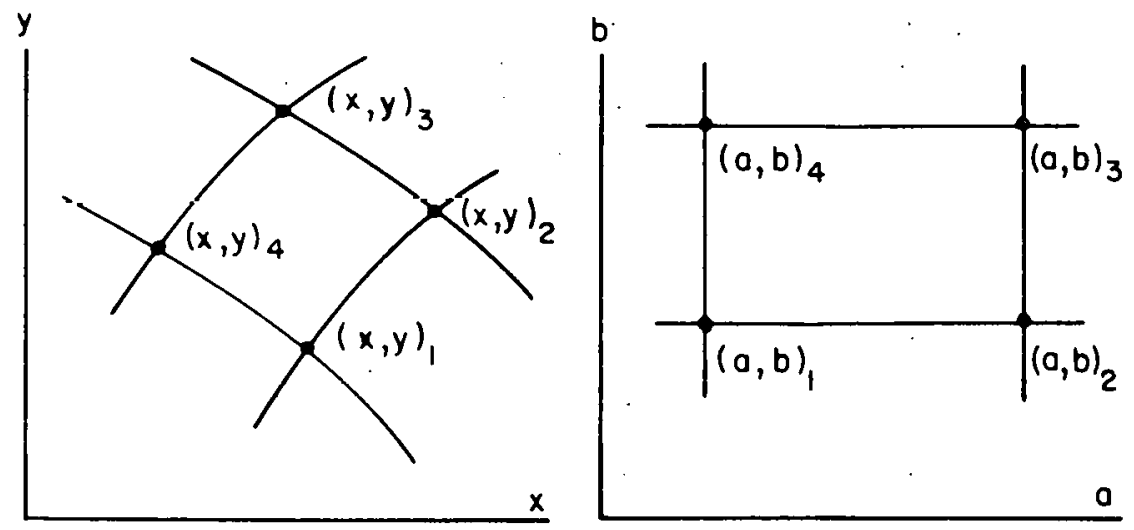

GLI-6312-3151

Fis. 3. The quadrilateral with vertices $(x, y)_{i} i=1,2,3,4$ in the $x, y$ plane $i$ the map (by the transformation $x=x(a, b), y \stackrel{1}{=} y(a, b)$ ) of the square in the $a, b$ plane. We take the square with its sides parallel to the $a$ and $b$ axes and with vertices $(a, b)_{i} i=1,2,3,4$. The point $(x, y)_{i}$ is the map of $(a, b)_{j}$ (i. e., $x_{i}=x\left(a_{i}, b_{i}\right)$ ctc.). 


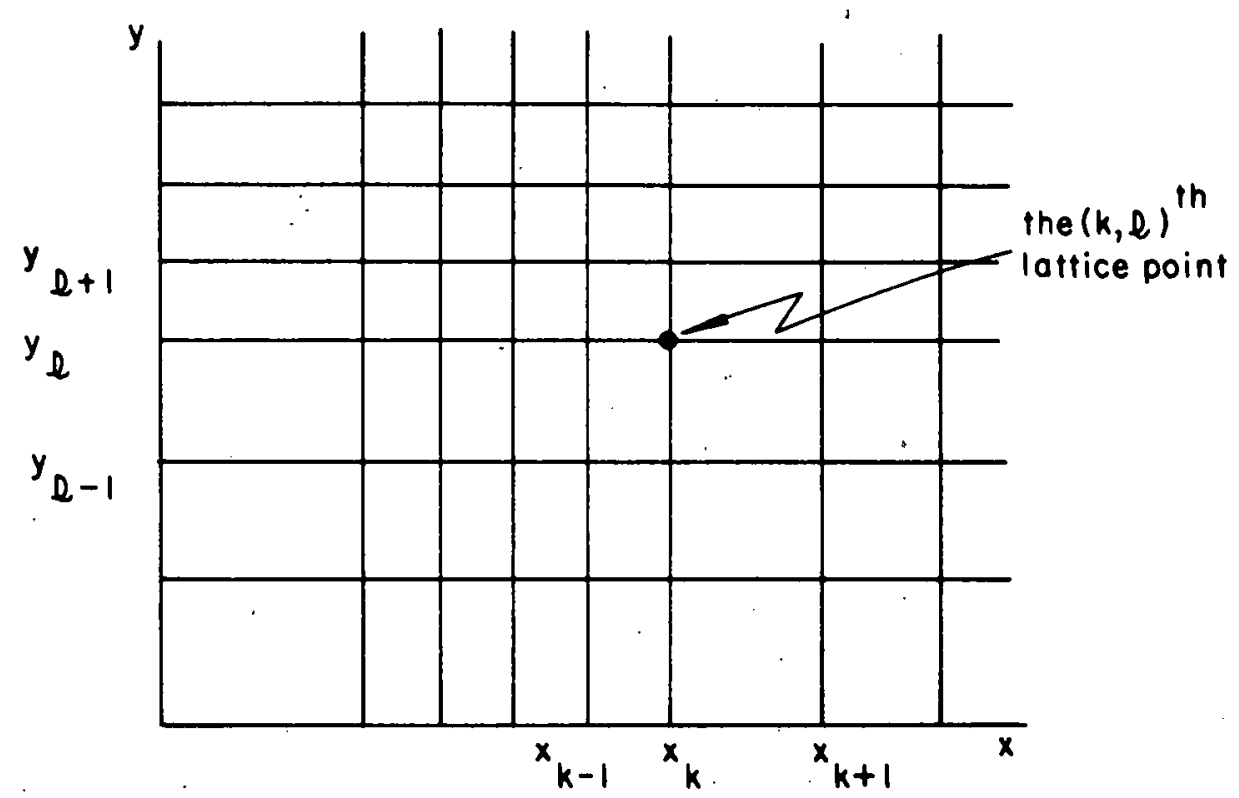

GLL-63IR-3I52

Fig. 4. A rectangular mesh defined by the straight lines $x_{k} k=0,1,2, \ldots$, $\mathrm{y}_{\ell} \ell=0,1,2, \ldots$ The lattice points of the mesh are the intersections $x=x_{k}$, $y^{\prime}=y_{\ell} k, \ell=0,1,2 \ldots$

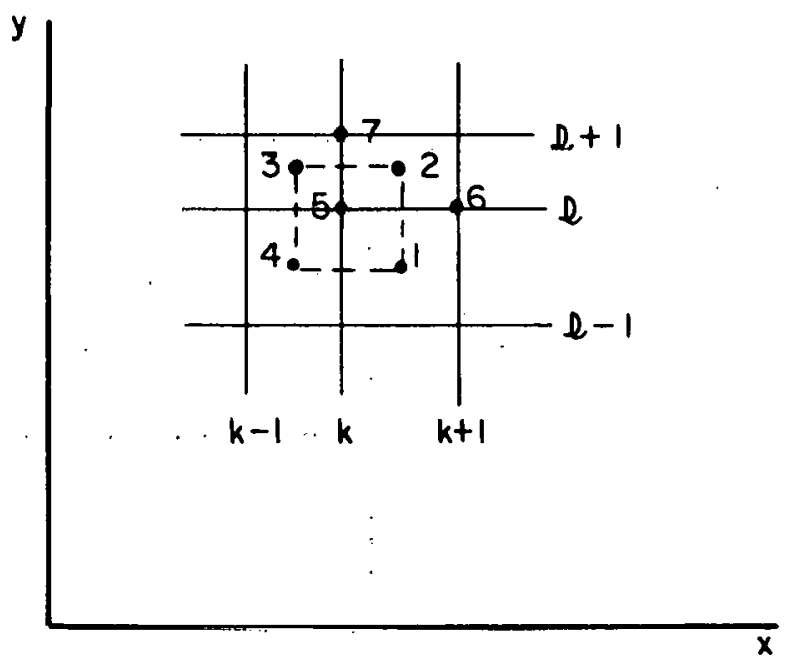

GLI $-6312-3153$

Fig. 5. Numbering used in Definition II. The dotted box with corners 1 , $2,3,4$ corresponds to a momentum cell. 


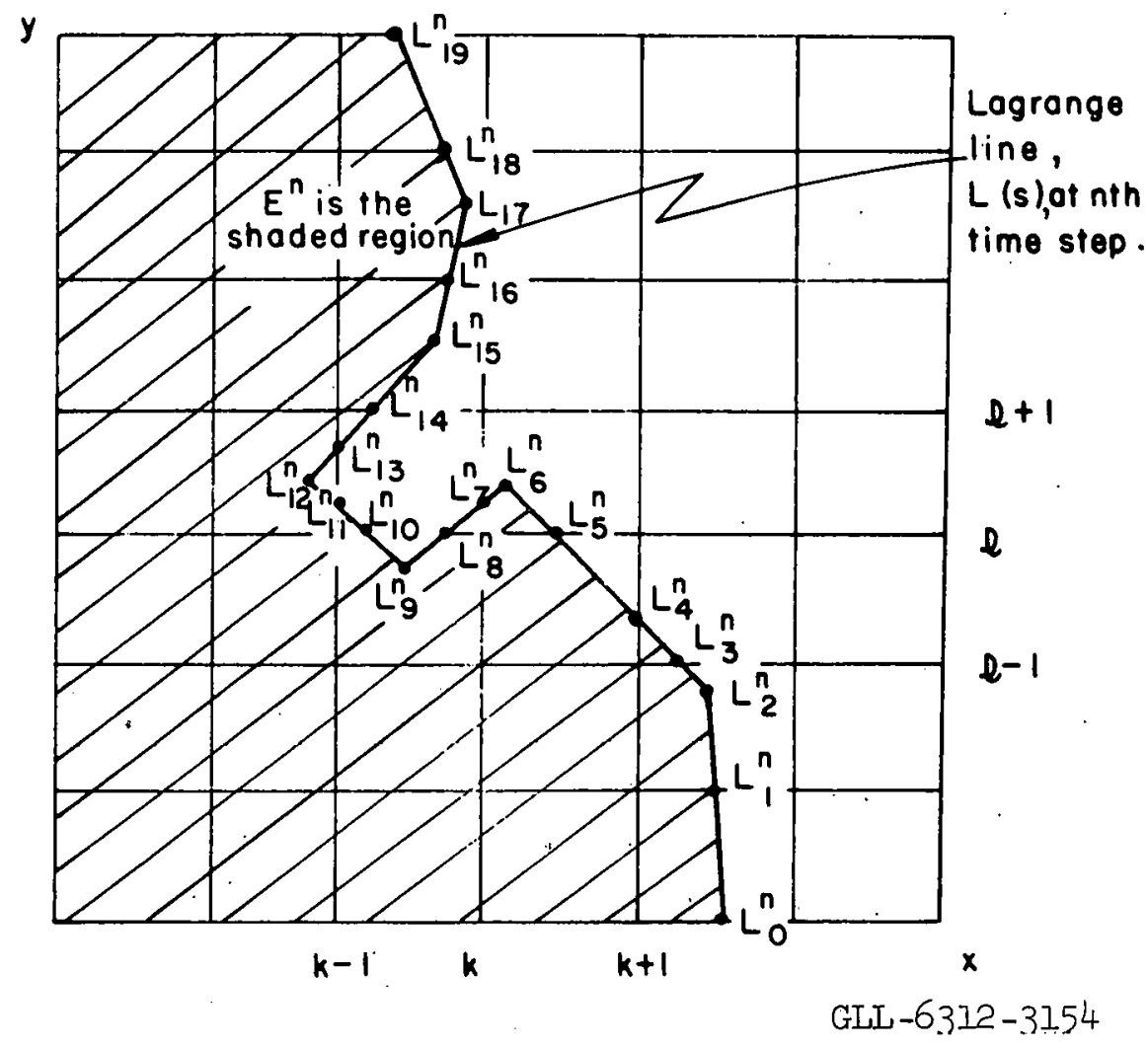

Fig. 6. An Eulerian region with a Lagrange boundary. (1) The polygonal line joining the points $L_{0}^{n}, L_{2}^{n}, L_{6}^{n}, L_{9}^{n}, L_{12}^{n}, L_{15}^{n}, L_{17}^{n}$, and $L_{19}^{n}$ is the Lagrange boumlary $L^{n}(s)$ at the nth time step. (2) The shadcd region including the boundary $L^{n}(s)$ is the Eulerian fluid region $E^{n}$. 


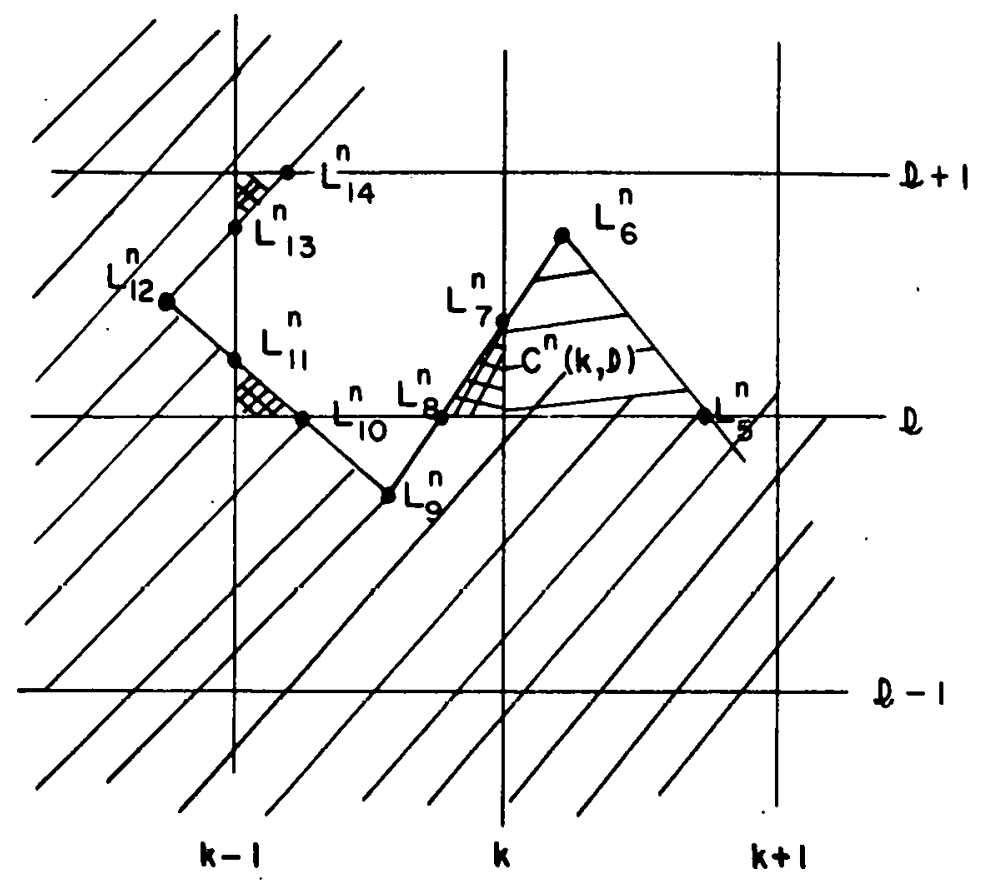

GLL $-6312-3155$

Fig. 7. An enlargement of the central portion of Fig. 6.

(1) $\partial C(k, l)$ is the polygon with vertices $L_{5}^{n}, L_{6}^{n}, L_{7}^{n}, G_{k, l}$ and there is one boundary sequence associated with $\partial C^{n}(k, l)$ which is

$$
\left\{V_{i}^{n}(k, \ell, 1)\right\}_{i=1,2, \ldots 5}=\left\{L_{5}^{n}, L_{6}^{n}, L_{7}^{n}, G_{k, \ell}, L_{5}^{n}\right\} \text {. }
$$

(2) $\partial C^{n 1}(k-1, l)$ is composed of three polygons with vertices (i) $\mathrm{L}_{7}^{n}, L_{8}^{n}, G_{k, l}$; (ii) $L_{10}^{n}, L_{11}^{n}, G_{k-1, \ell}$; (iii) $L_{13}^{11}, L_{14}^{n}, G_{k-1, \ell+1}$, and there are three boundary sequences associated with $\partial C^{\mathrm{n}}(\mathrm{k}-1, \ell)$, namely,

$$
\begin{aligned}
& \left\{V_{i}^{n}(k-1, l, 1)\right\}_{i=1,2,3,4}=\left\{L_{7}^{n}, L_{8}^{n}, G_{k, l}, L_{7}^{n}\right\} \\
& \left\{V_{i}^{n}(k-1, l, 2)\right\}_{i=1,2, \ldots 4}=\left\{L_{10}, I_{1}, r_{k-1, l}, L_{1} n\right\} \\
& \left\{V_{i}^{n}(k-1, l, 3)\right\}_{i=1,2, \ldots 4}=\left\{L_{13}, L_{14}, G_{k-1, l+1}, L_{13}\right\} .
\end{aligned}
$$

(3) $\partial C^{n}(k-1, \ell-1)$ is the polygon with vertices $L_{8}^{n}, L_{9}^{n}, L_{10}, G_{k-1, \ell}$,

$G_{k-1, \ell-1}, G_{k, \ell-1}, G_{k, \ell}$ and has one boundary sequence associated with it namely $\left\{v_{i}^{n}(k-1, \ell-1,1)\right\}_{i=1.2, \ldots 8}=\left\{L_{8}^{n}, L_{9}^{n}, L_{10}^{n}, G_{k-1, \ell}, G_{k-1, \ell-1}, G_{k, l-1}, G_{k, \ell}, L_{8}^{n}\right\}$. 


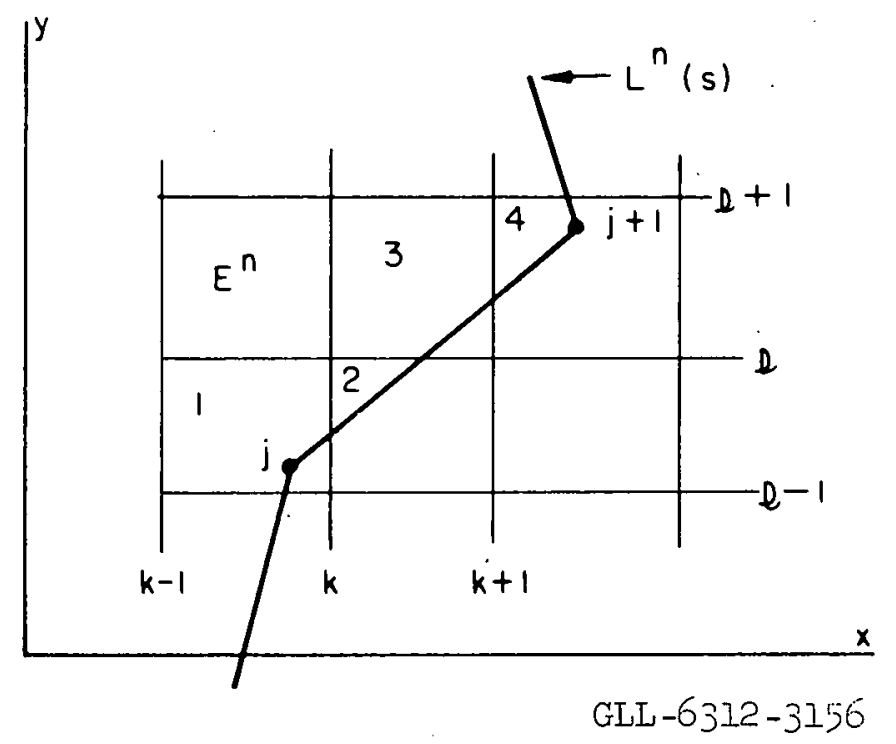

Fig. 8. The pressure at the Lagrange-Eulerian interface is determined from the Eulerian mesh. The Lagrange line segment joining $j$ and $j+l$ forms part of the bounderies of zones 1, 2, 3, and 4. Hence,

$$
P_{j+1 / 2}^{n}=\frac{\sum_{i=1}^{4} P_{i}^{n} J_{i}^{n}}{\sum_{i=1}^{4} J_{i}^{n}} .
$$

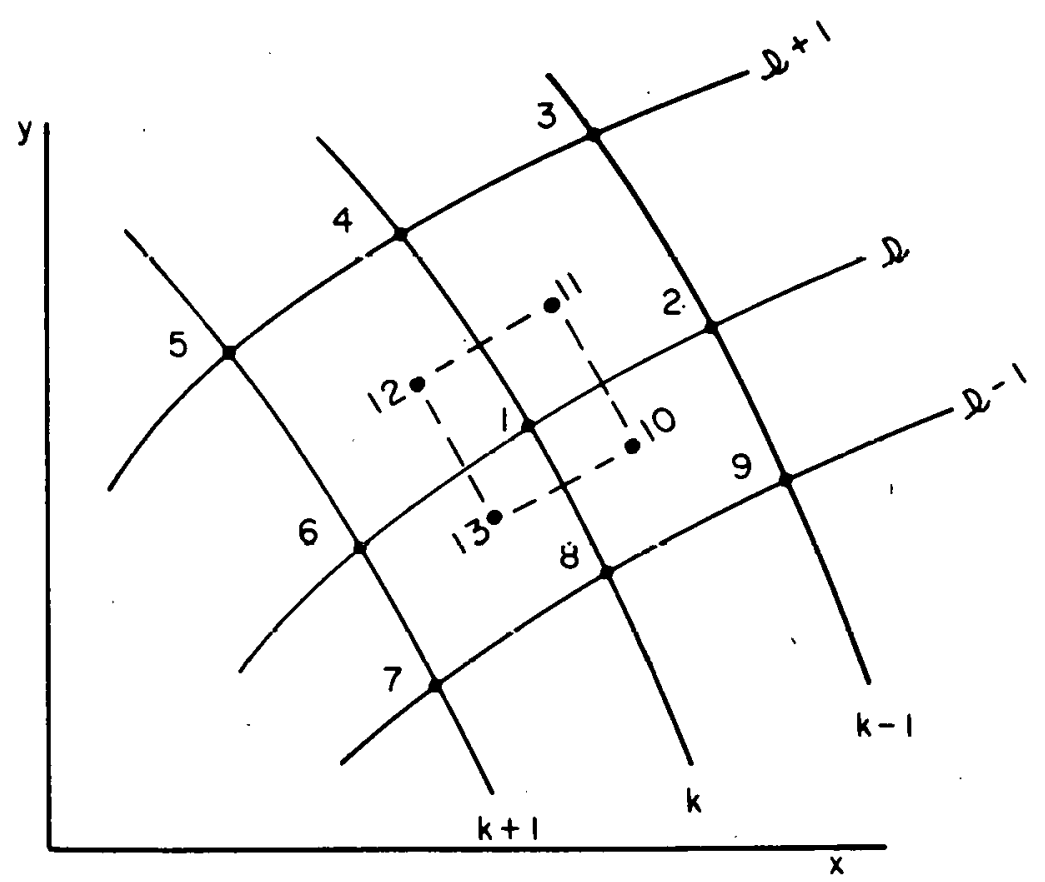

GLL-6312-3157

Fig. 9. Numbering used in Definition III. 


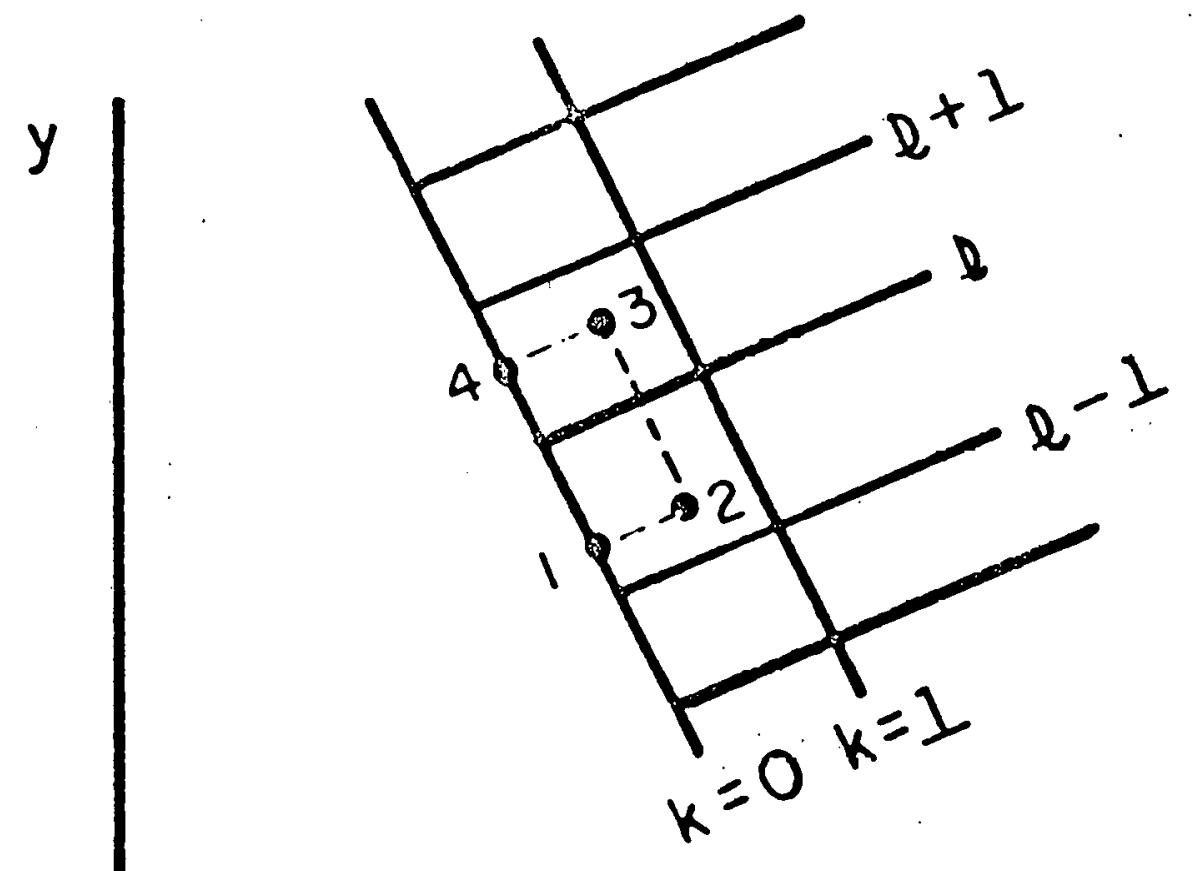

Fig. 10. Velocity calculation at the Lagrangc boundary $k=0$. The pressure is supposed known along the Lagrange line $\mathrm{k}=0$ and then by (9) and (10)

$$
\begin{aligned}
& \left(\frac{1}{\mu} P_{x}\right)_{0, \ell}^{n}=\frac{\sum_{i=1}^{i=4}\left(\frac{P_{i+1}^{n}+P_{i}^{n}}{2}\right)\left(y_{1+1}^{n}-y_{i}^{n}\right)}{\frac{1}{4}\left[(\rho J)_{2}^{n}+(\rho J)_{3}^{n}\right]} . \\
& \left(\frac{1}{\rho} P_{y}\right)_{0, \ell}^{n}=\frac{-\sum_{i=1}^{i=4}\left(\frac{P_{i+1}^{n}+P_{i}^{n}}{2}\right)\left(x_{i+1}^{n}-x_{i}^{n}\right)}{\frac{1}{4}\left[(\rho J)_{2}^{n}+(\rho J)_{3}^{n}\right]} .
\end{aligned}
$$




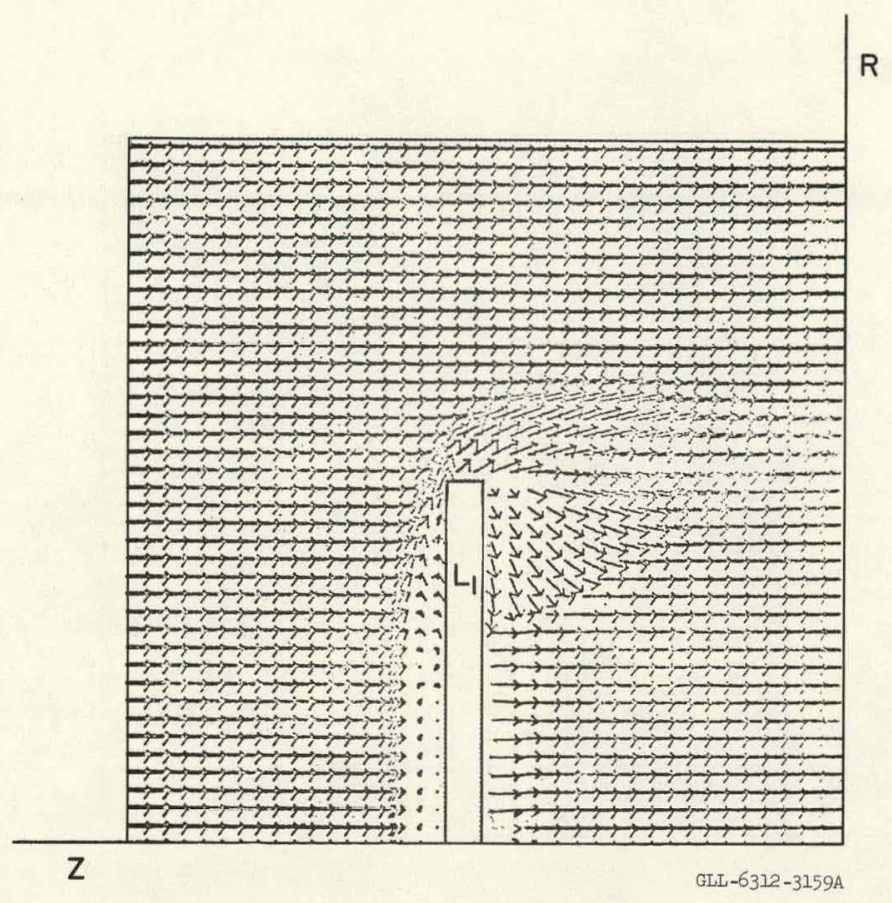

Fig. 11. Cathode-ray tube plot of Mach $\infty$ flow of a perfect gas past a rigid disk. The disk is represented by the fixed Lagrange grid $L_{1}$. (a) Shortly after $t=0$ the shocked gas begins to expand into the cavitated region behind the disk. The bow shock is becoming established and begins to move out.

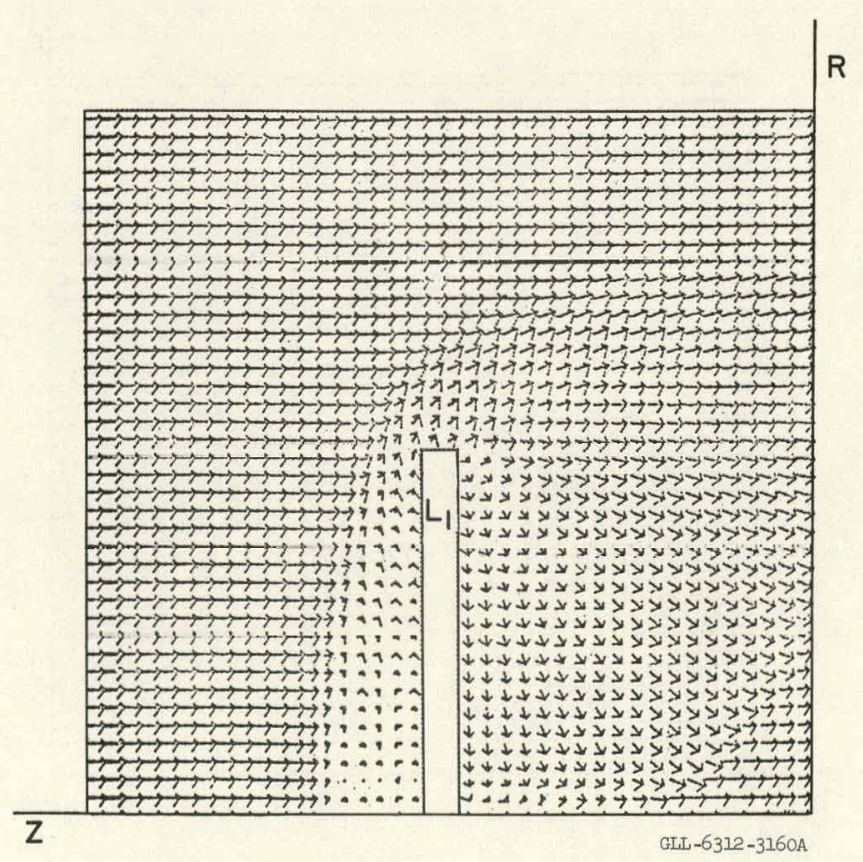

Fig. 11. (b) The bow shock continues to move out. 


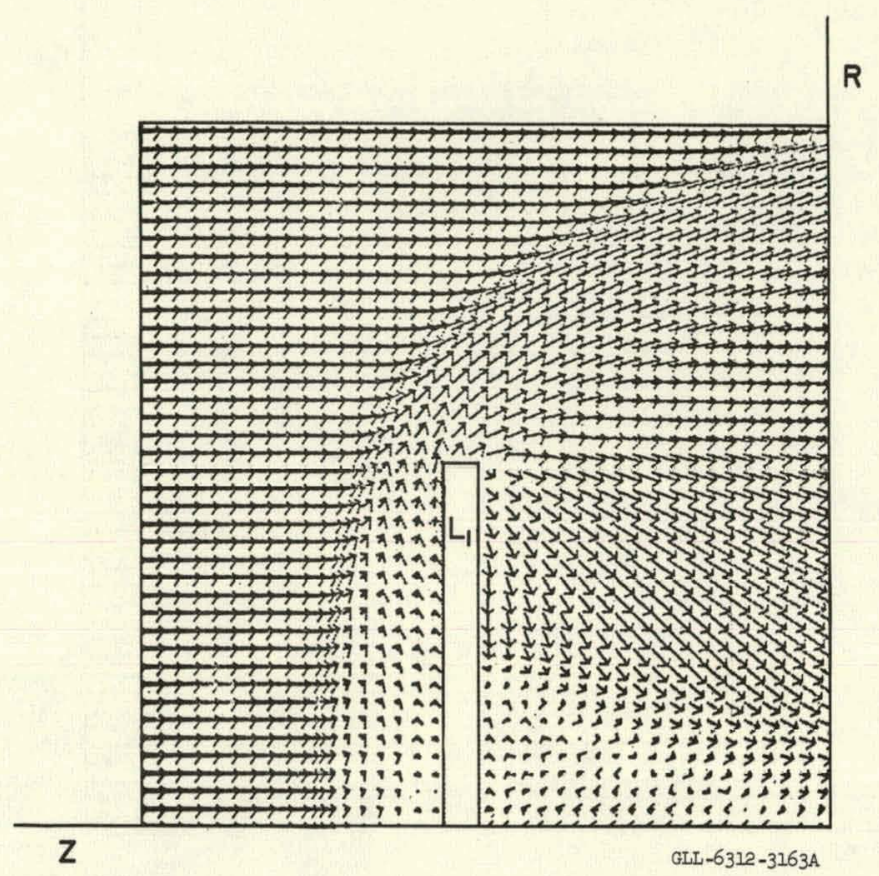

Fig. 11. (c) Flow behind the disk begins to stagnate on the axis.

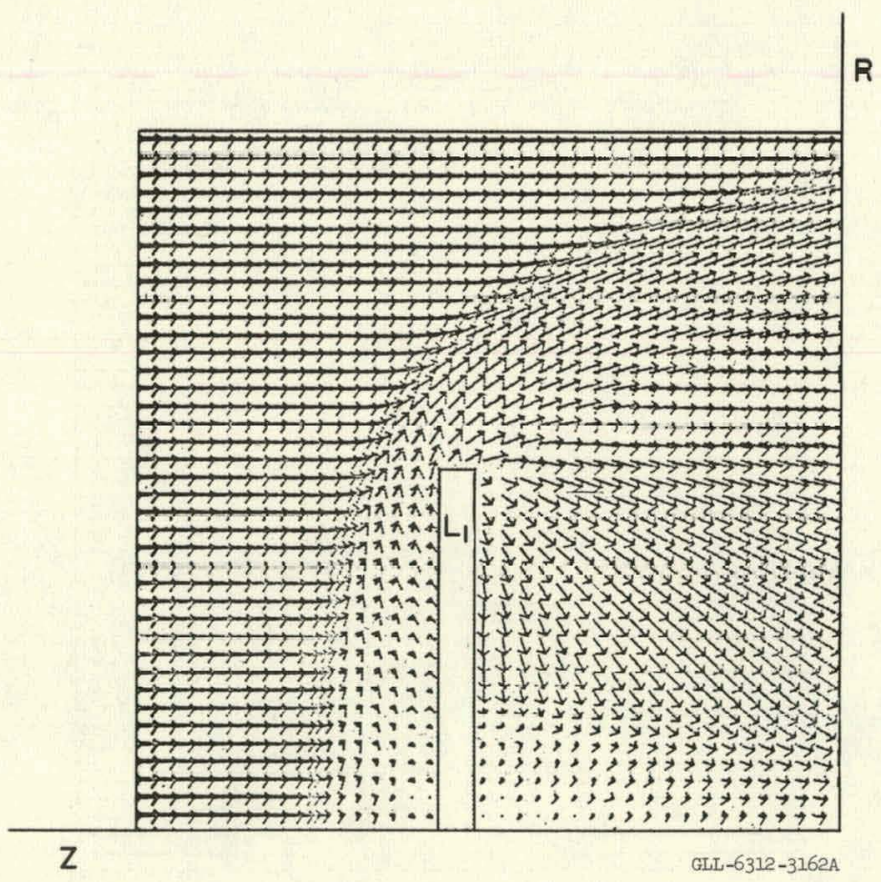

Fig. 11. (d) The stagnation region behind the disk has become more extended. 


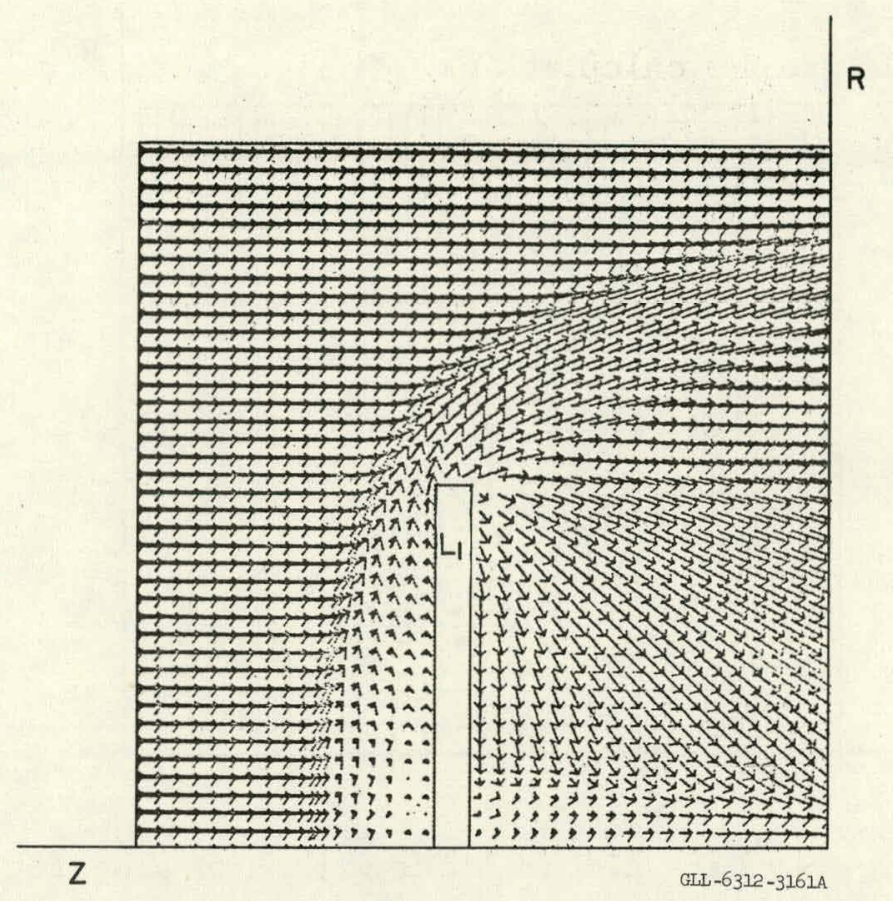

Fig. 11. (e) A slow circulation begins in the stagnation region.

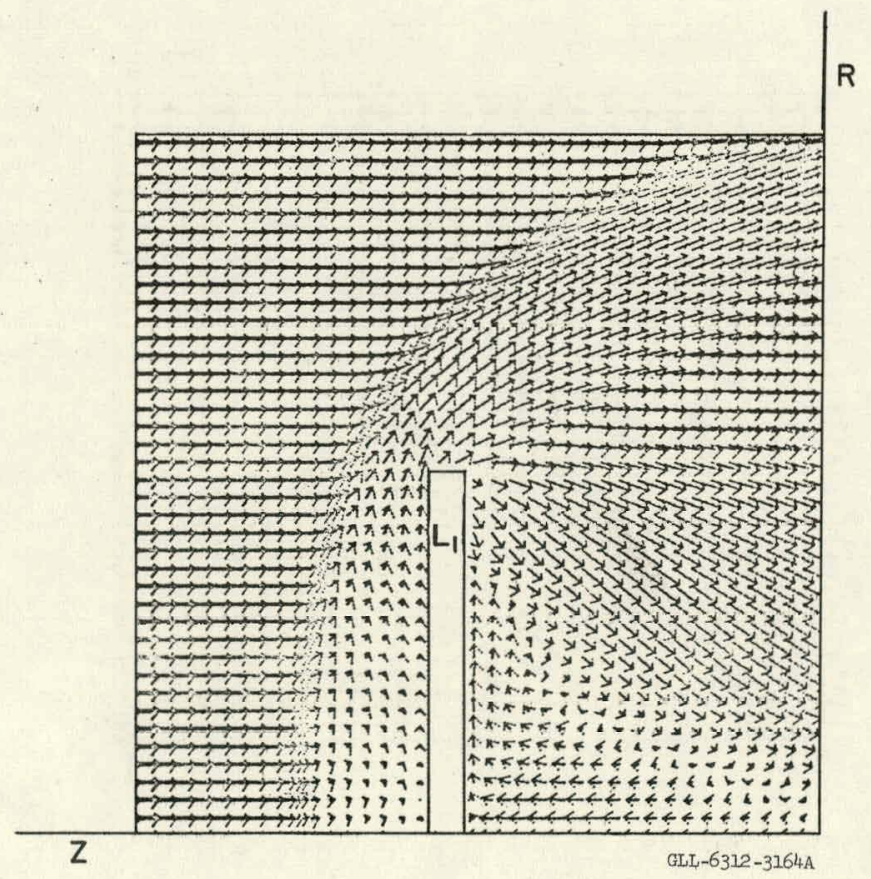

Fig. 11. (f) This is essentially the steady-state configuration; we see that a slow circulation behind the disk has developed. 


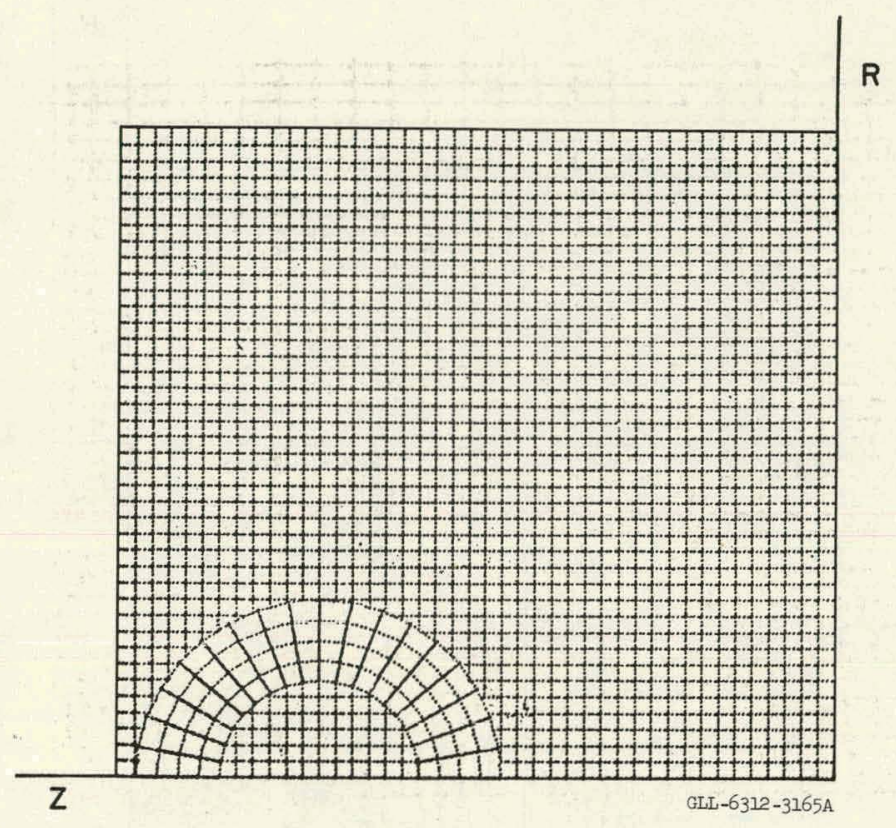

Fig. 12. Cathode-ray tube plot of Mach $\infty$ flow of a perfect gas past a dense (but compressible) metal sphere. (a) Initial configuration. The sphere is approximated by both the Lagrange grid and (the central portion of the sphere) by the Eulerian mesh. The Eulerian region outside of the sphere approximates the gas.

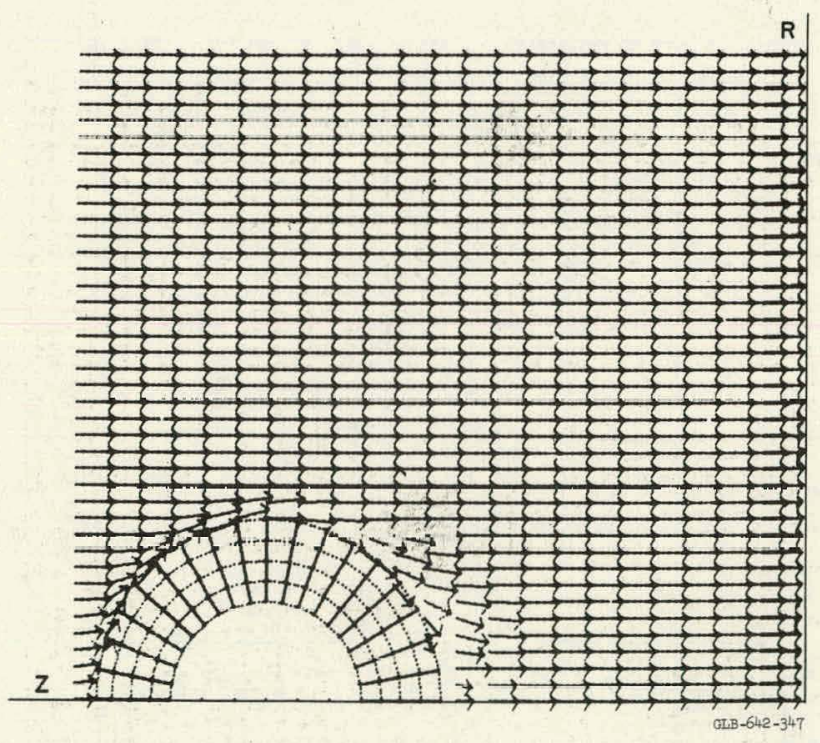

Fig. 12. (b) The flow of gas over the sphere shortly after $t=0$. The initial conditions of the gas are zero temperature (hence zero sound speed) and a uniform velocity parallel to the $Z$ axis. The compressible sphere is initially at rest and at zero temperature. The gas velocities are plotted only at every other vertical line in the Eulerian mesh. 


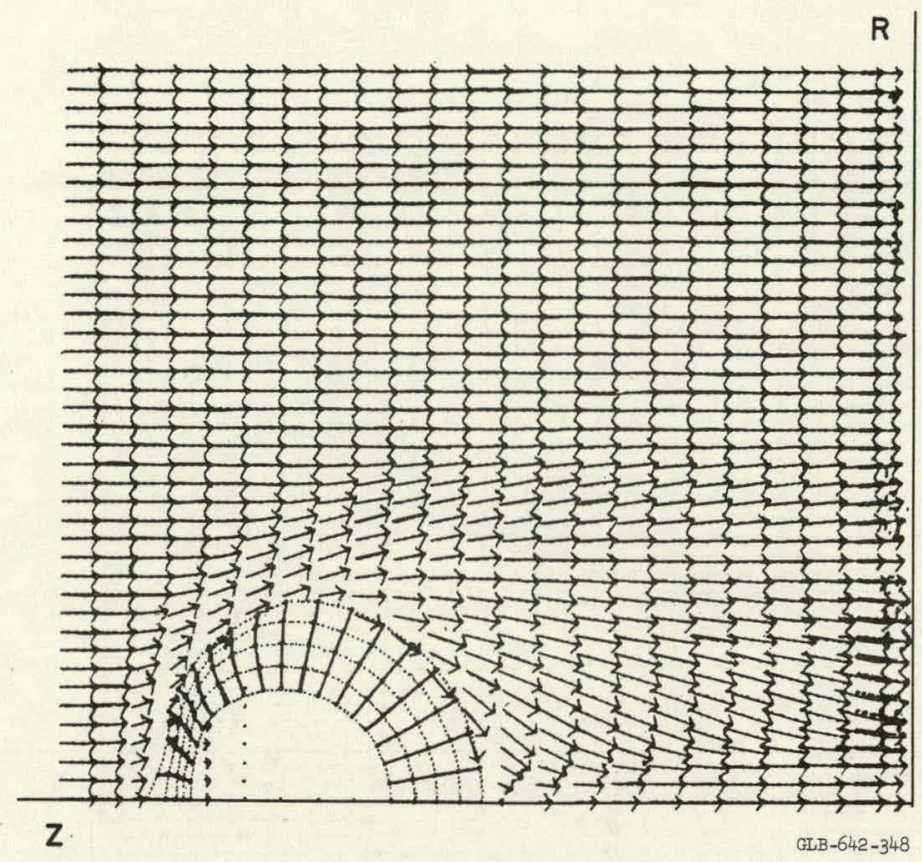

Fig. 12. (c) The bow shock begins to form and the sphere compresses as the transmitted shock proceeds into the sphere. Here the gas velocities are plotted at every other Eulerian mesh point. We see velocities appearing in the Eulerian region of the sphere.

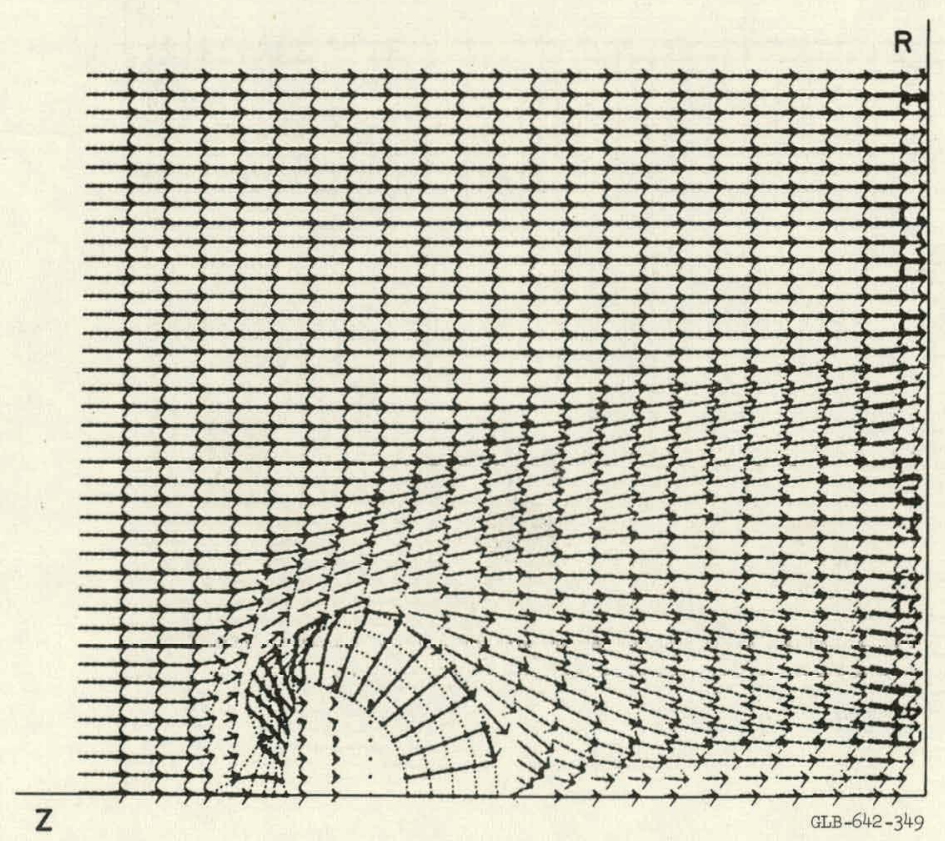

Fig. 12. (d) In the start-up of the problem a slight dent was produced on the leading edge of the sphere. Since the flow is Taylor unstable, this perturbation grows with time, and we see a spike and a bubble forming. 


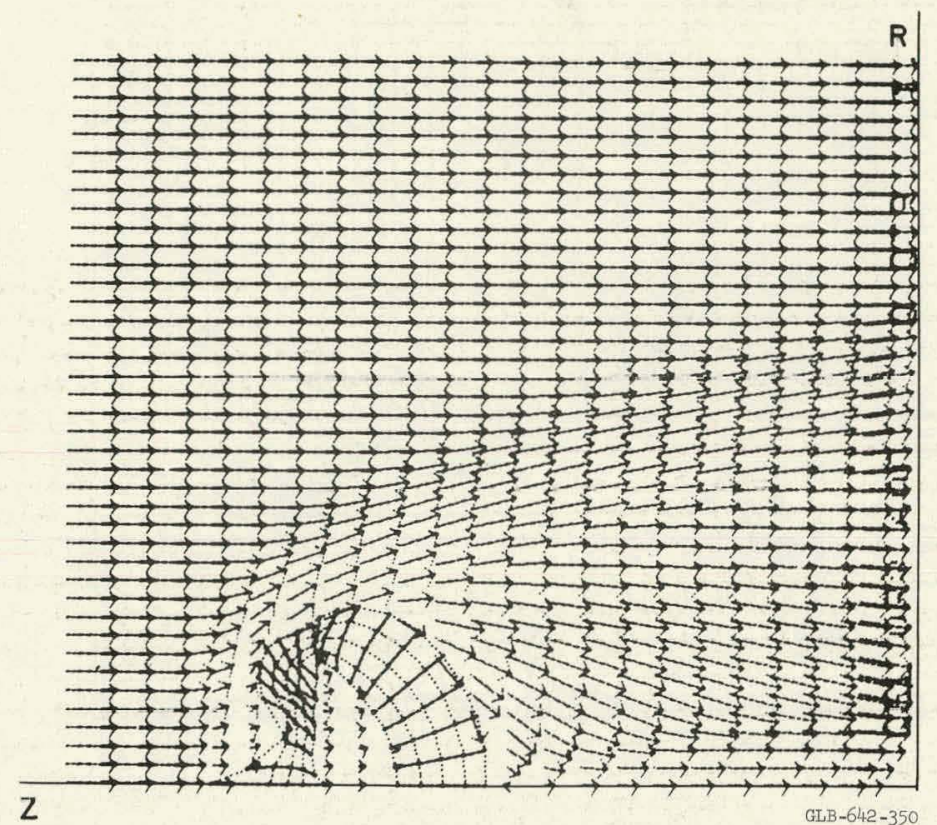

Fig. 12. (e) The transmitted shock has progressed more than halfway through the sphere. The instability on the leading edge has become more pronounced.

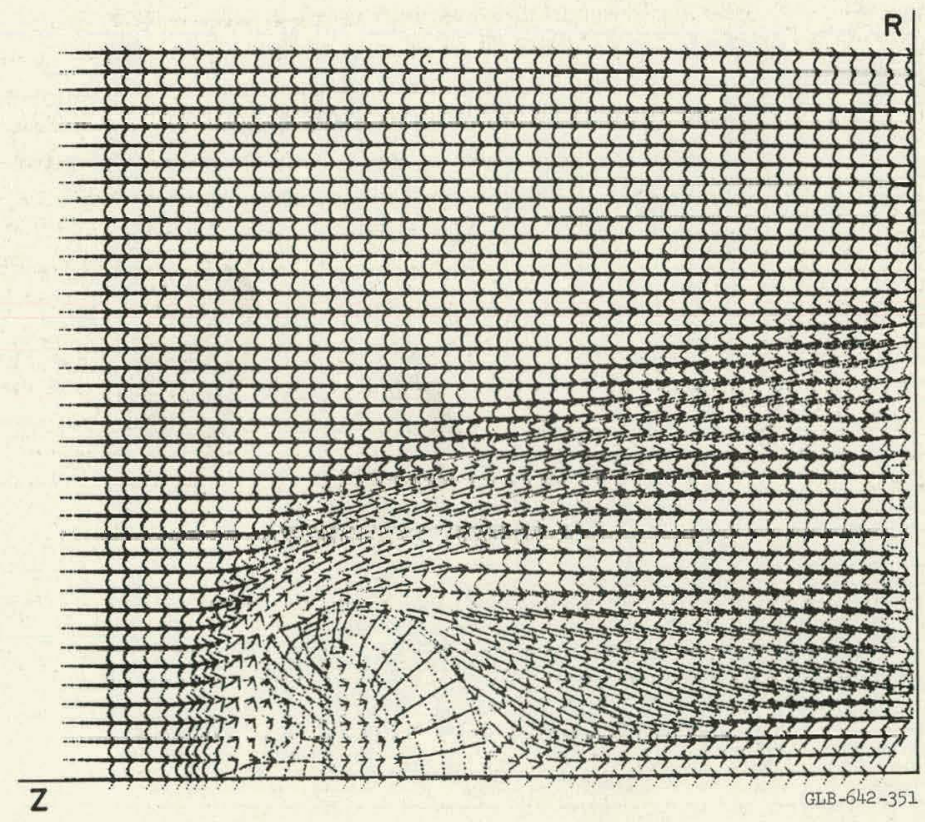

Fig. 12. (f) Here we begin to plot the gas velocities at every Eulerian mesh point. 


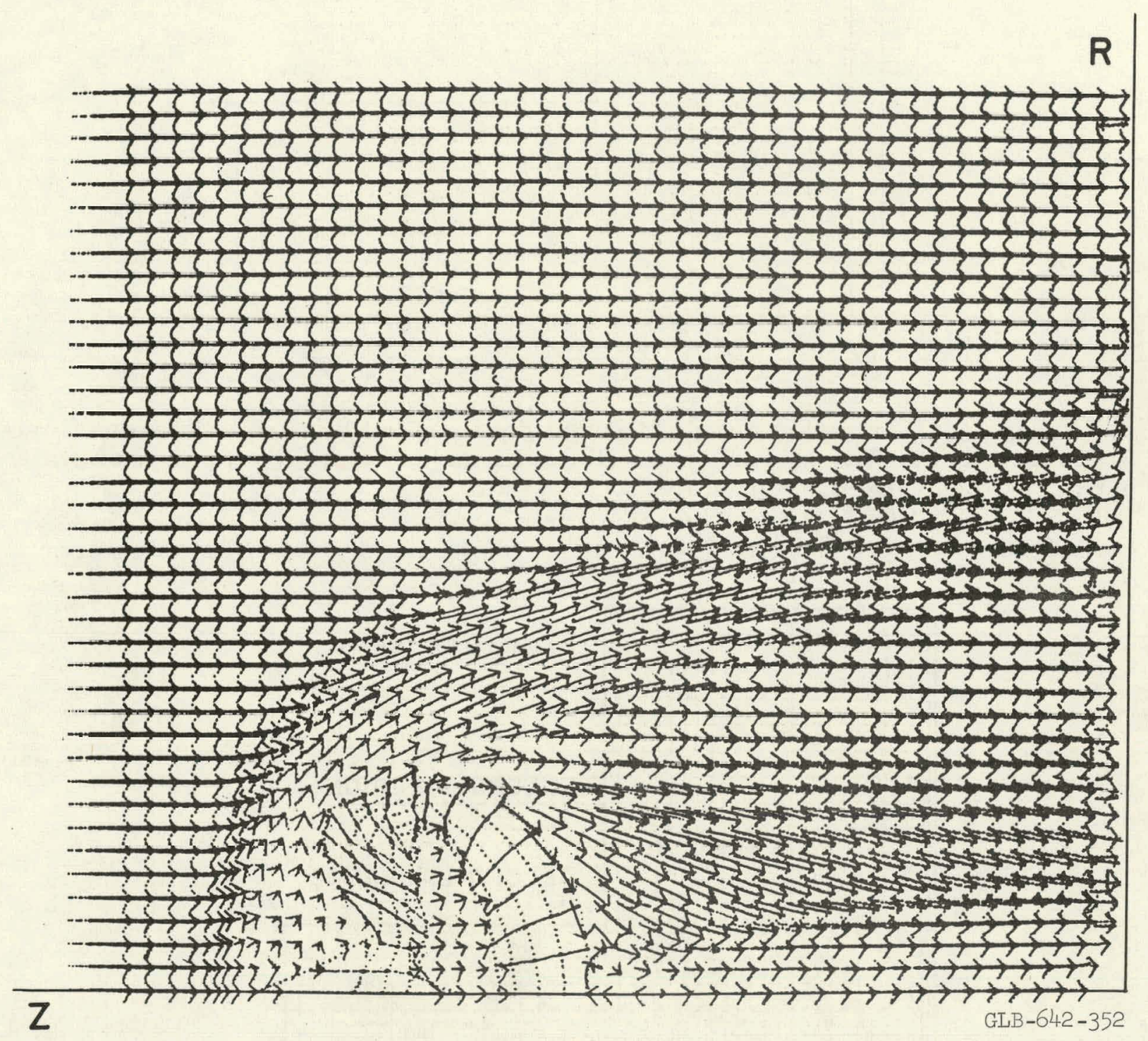

Fig. 12. (g) We see that the amplitude has progressed beyond the Eulerian region of the sphere. The problem was discontinued at this time. 


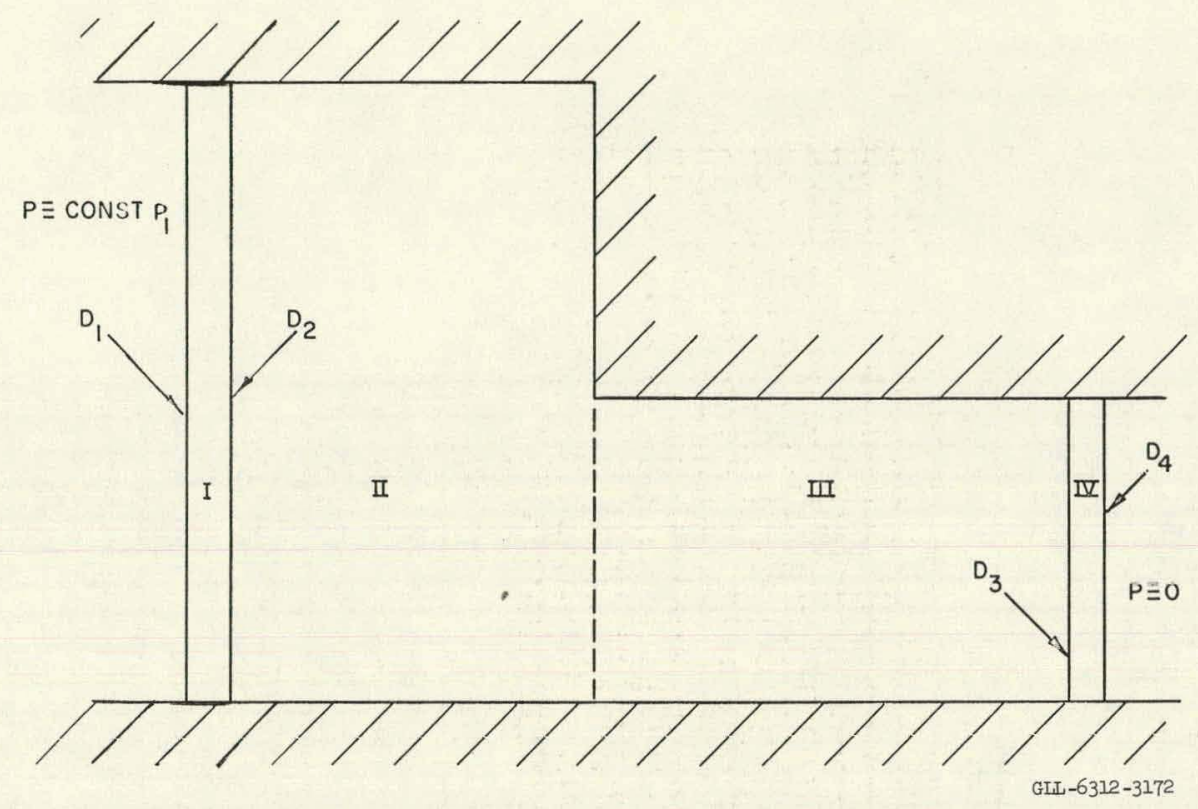

Fig. 13. (a) Here we consider a region occupied by three different fluids: Regions I, II and III, IV. Regions II and III have the same equation of state but correspond to different states of the fluid.

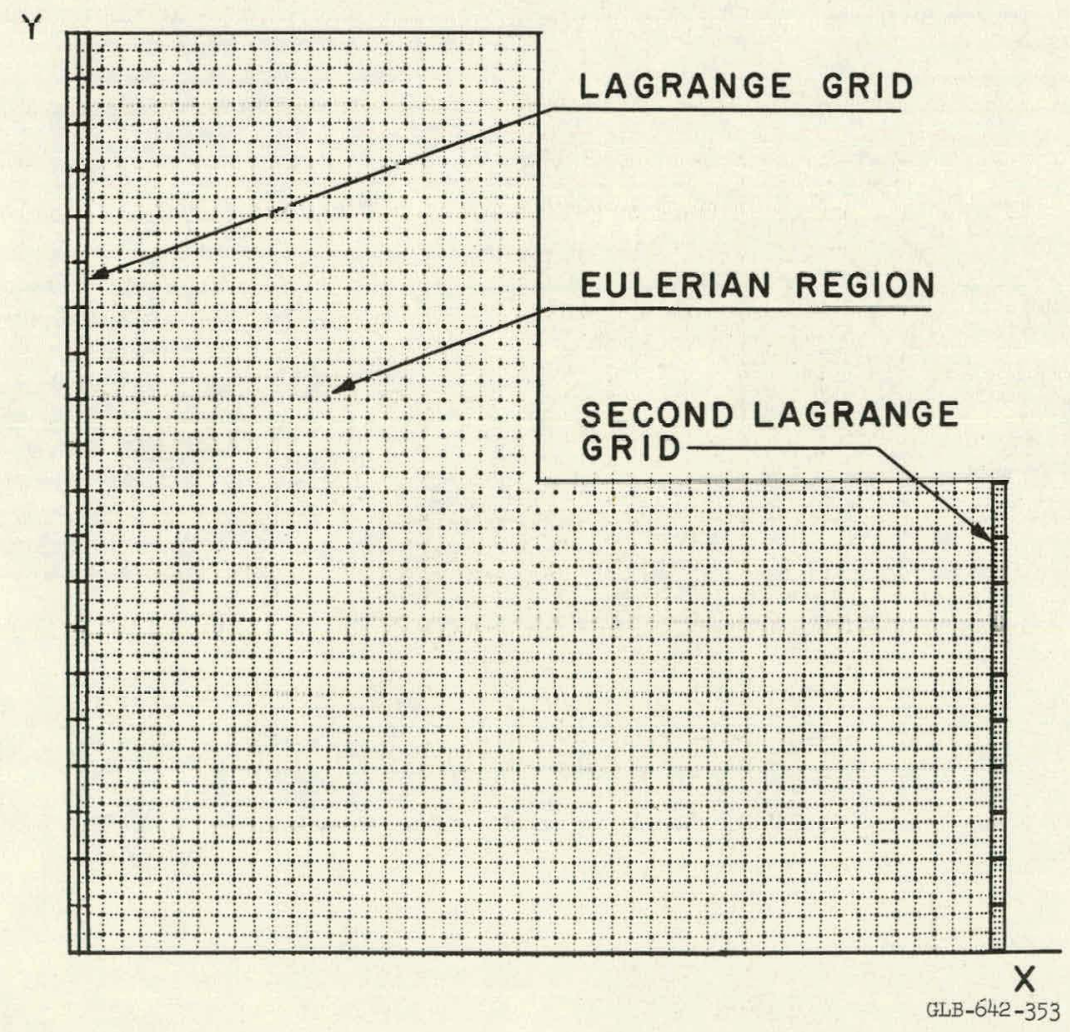

Fig. 13. (b) The initial position of the two Lagrange grids and the Eulerian mesh. 


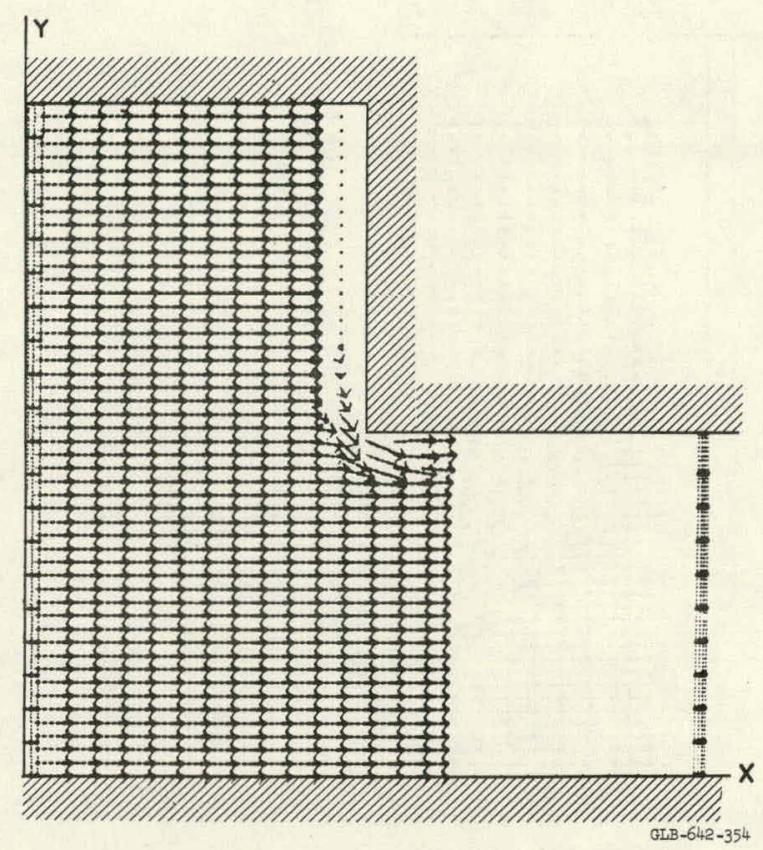

Fig. 13. (c) Shortly after $t=0$ : A reflected shock is developing and the initial shock continues toward the second Lagrange piston.

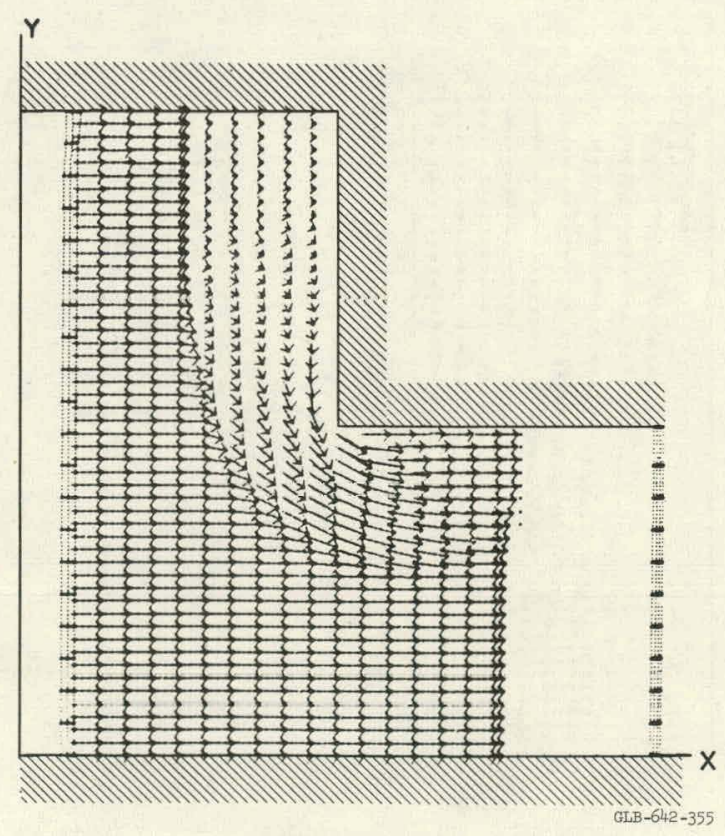

Fig. 13. (d) The reflected shock produces a high pressure region, which produces flow around the corners. As a result of this flow the shock that proceeds to the right is no longer plane. 


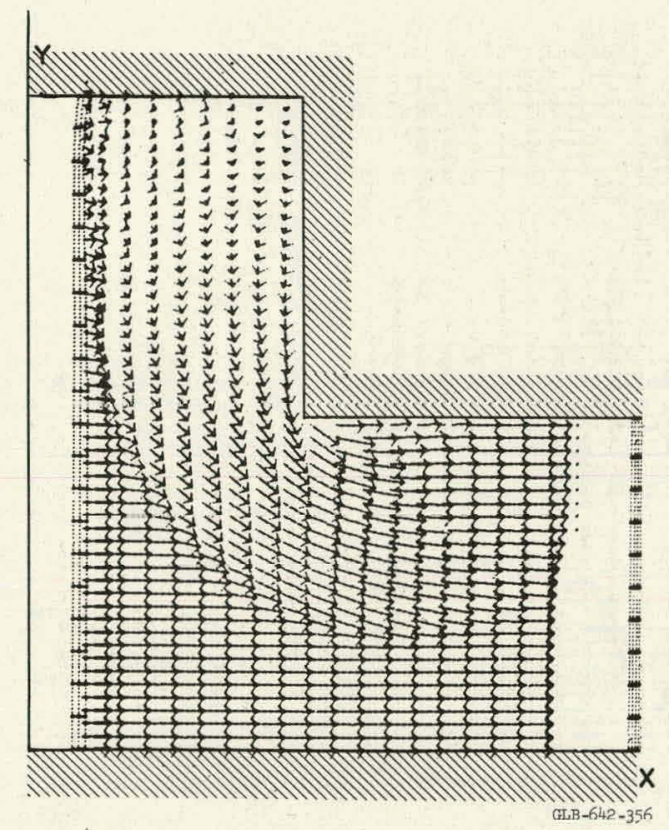

Fig. 13. (e) The reflected shock has reached the upper portion of the piston and has begun to turn it around.

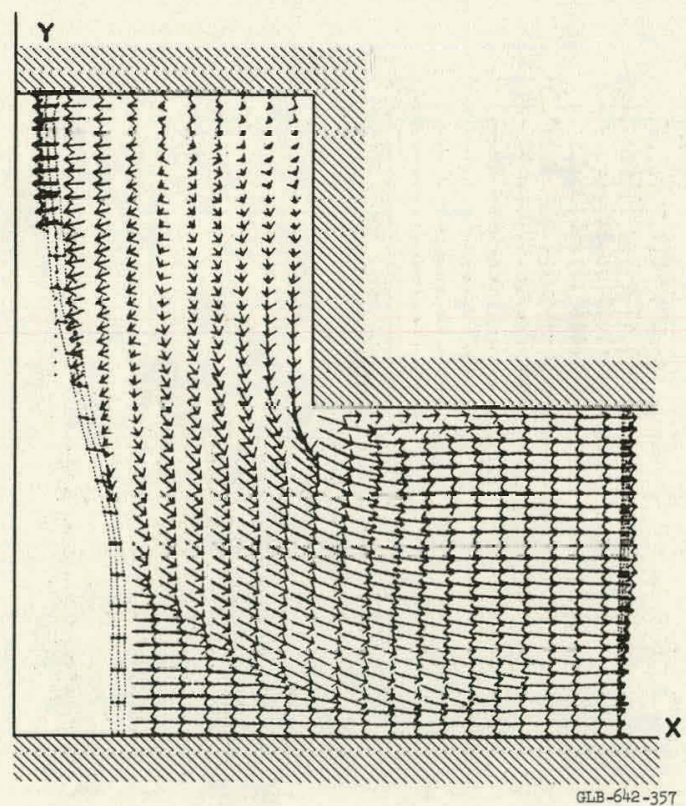

Fig. 13. (f) The reflected shock has turned the upper portion of the lefthand piston around. The main shock has reached the second piston and a transmitted shock proceeds into it. 


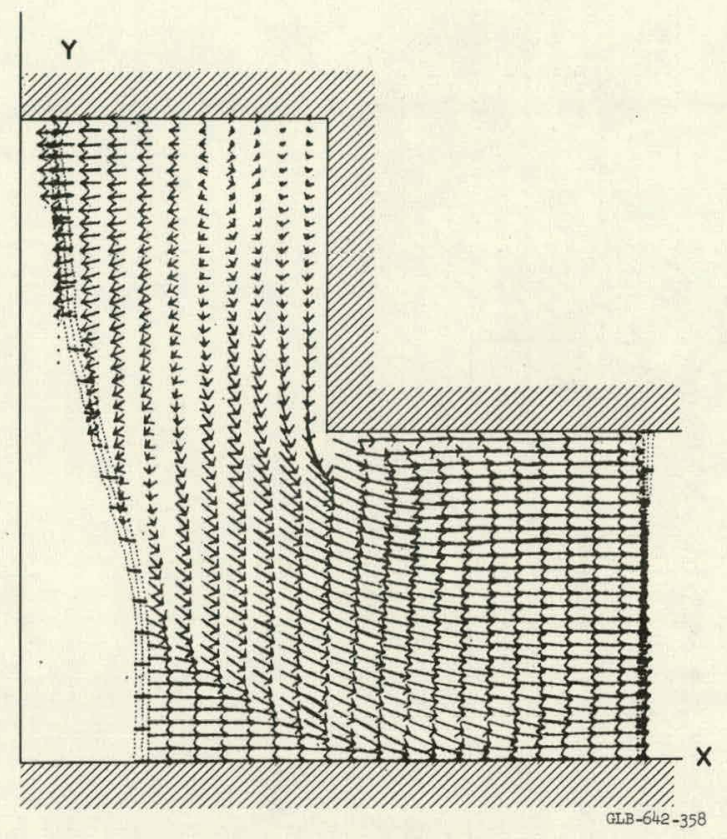

Fig. 13. (g) The shock is through the upper half of the second piston and the free surface has begun to expand.

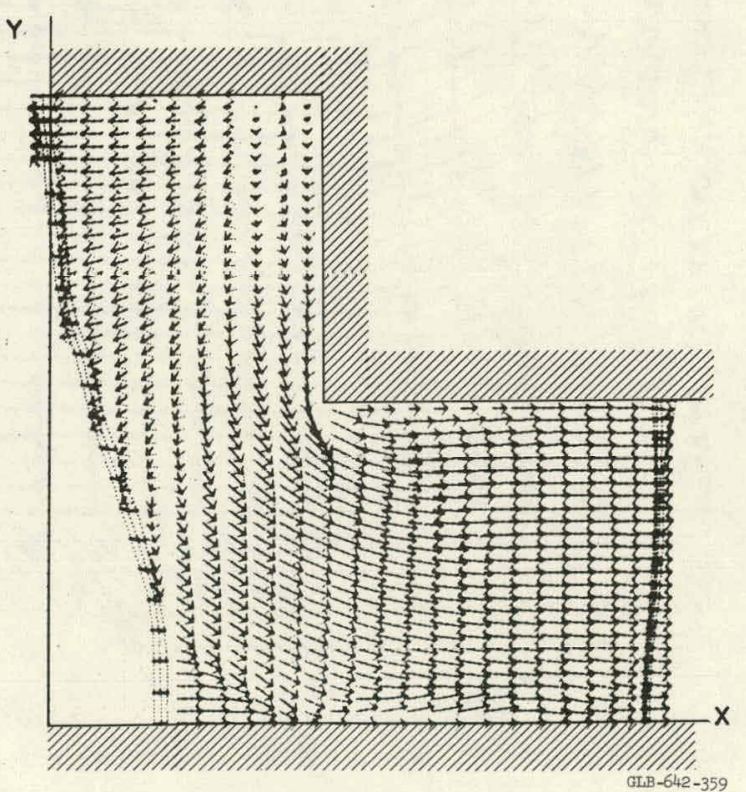

Fig. 13. (h) The flow around the corner has reached the $x$ axis and is deflected. 


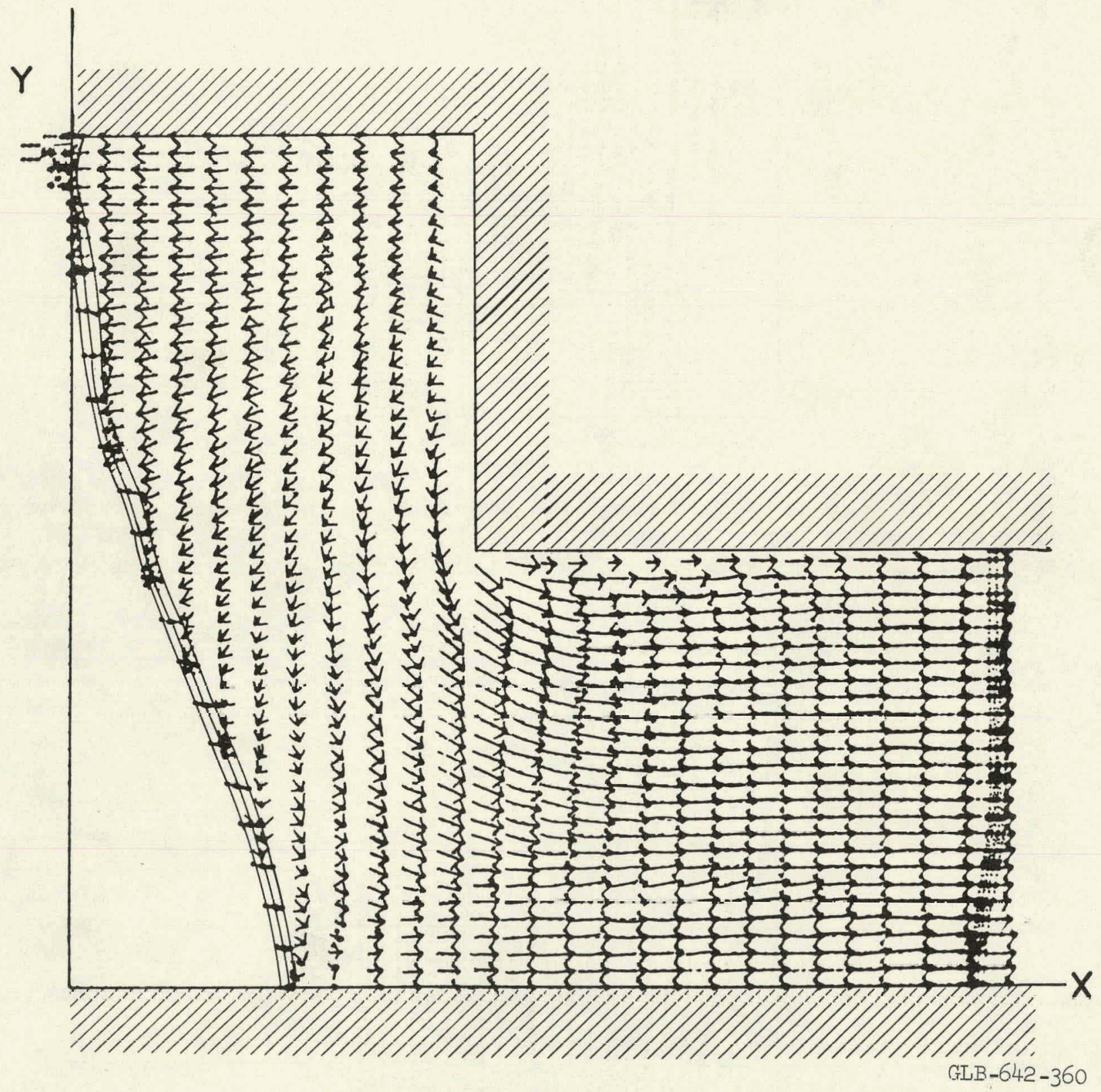

Fig. 13. (i) The shock on the axis has now stopped the forward motion of the first piston. The calculation was discontinued at this time. 